\title{
RIEMANN SURFACES AND THEIR ASSOCIATED WIRTINGER VARIETIES
}

\author{
BY R. C. GUNNING
}

1. The canonical imbedding of a compact Riemann surface in its associated Jacobi variety, an algebraic complex torus, has long been of fundamental importance in the study of Riemann surfaces, and the basic holomorphic functions that arise in the study of algebraic tori, the theta functions, naturally play an essential role in this part of the theory. The central role and essential properties of the simple theta function in the study of Riemann surfaces were established by Riemann and lie at the core of the subject. Some of the basic properties of higher-order theta functions on general algebraic tori, principally those properties involved in the projective imbedding of these tori, were established by Kummer for two-dimensional tori and extended by Wirtinger to higher-dimensional tori, and investigations in this direction have been pursued fairly steadily ever since. Some quite deep and subtile properties of the higher-order theta functions of Jacobi varieties, as distinct from general algebraic tori, were established by Frobenius and Schottky, and investigations in this direction have also been pursued ever since, perhaps somewhat fitfully and implicitly at the beginning (since the higher-order theta functions can be expressed as various products of translates of simple theta functions and thereby disguised, sometimes conveniently and sometimes confusingly) but with increasing vigor, particularly in the last decade. Part of this current activity, particularly among algebraic geometers, derives from Fay's stimulating book on theta functions and Riemann surfaces, while another part no doubt derives from the role that theta functions of Jacobi varieties have been discovered to play in providing explicit solutions of some important nonlinear partial differential equations. Both of these inspirations have led to fascinating new approaches to and results about Schottky's problem of characterizing Jacobi varieties among more general algebraic tori; there has thus been a surprising revival of interest in this once obscure but always intriguing topic.

The aim of the present paper is to provide a survey of some of this recent activity that most directly involves the study of Riemann surfaces itself, together with a discussion of a few new results in the directions surveyed. The

An expanded version of an invited address presented at the summer meeting of the American Mathematical Society at Albany, New York on August 8, 1983; received by the editors February 14, 1984.

1980 Mathematics Subject Classification. Primary 14K25; Secondary 14K20, 14 H40. 
motif is the role of theta functions in describing various subvarieties of Jacobi varieties that are of fundamental importance in the study of Riemann surfaces. Many of these subvarieties can be described quite simply and explicitly in terms of the image of the Jacobi variety under the mapping into projective space given by a basis of the second-order theta functions; the image variety is the Wirtinger variety of the title, a natural generalization of the Kummer surface. The hope is that this will at least in part provide an introduction to this topic, indicating why the questions considered are of interest and how the current investigations follow naturally in the direction of Riemann's earlier work. Present knowledge of the finer properties of the higher-order theta functions of Jacobi varieties is still quite limited, many results clearly having a rather preliminary nature. A further hope is that this will also indicate one point of view of the current state of affairs in this area and a number of very promising questions for further investigation. It should be emphasized that this is a rather limited survey, and is not intended to cover the many other approaches to the Schottky problem or the extensive recent work on theta functions and partial differential equations.

2. Let $M$ be a compact connected Riemann surface of genus $g>0$ and choose a fixed base point $p_{0} \in M$ and a set of canonical generators $\alpha_{1}, \ldots, \alpha_{g}, \beta_{1}, \ldots, \beta_{g}$ of the fundamental group $\pi_{1}\left(M, p_{0}\right)$; topologically the surface $M$ is just a sphere with $g$ handles, and $\alpha_{j}, \beta_{j}$ can be considered as a pair of paths around the $j$ th handle. The $2 g$ cycles carried by these paths are free generators of the homology group $H_{1}(M, \mathbb{Z})$, and will also be denoted by $\alpha_{j}, \beta_{j}$. Also choose a basis $\omega_{1}, \ldots, \omega_{g}$ for the complex vector space of holomorphic differential one-forms or Abelian differentials on $M$; these are all closed differential forms, and the $g \times 2 g$ matrix consisting of the integrals or periods of the differentials $\omega_{i}$ along the cycles $\alpha_{j}, \beta_{j}$ is called the period matrix for the surface $M$ in terms of the choices made for the paths $\alpha_{j}, \beta_{j}$ and for the basis $\omega_{i}$. To be more explicit let $\omega$ denote the column vector of length $g$ formed of the differentials $\omega_{i}$, so that its transpose is the row vector ${ }^{t} \omega=\left(\omega_{1}, \ldots, \omega_{g}\right)$, and define the period matrix to be

$$
\Lambda=\left(\int_{\alpha_{1}} \omega, \ldots, \int_{\alpha_{g}} \omega, \int_{\beta_{1}} \omega, \ldots, \int_{\beta_{g}} \omega\right) .
$$

It is always possible to choose the basis $\omega_{i}$ so that the period matrix has the form $\Lambda=(I, \Omega)$, where $I$ is the $g \times g$ identity matrix and $\Omega$ is an element of the Siegel upper half-space $\mathfrak{S}_{g}$, the cone of $g \times g$ complex symmetric matrices having positive definite imaginary parts; such a choice will henceforth always be supposed made.

The $2 g$ columns of the matrix $\Lambda$ are always linearly independent over the real numbers; thus when $C^{g}$ is viewed as the real vector space $\mathbb{R}^{2 g}$ the columns of $\Lambda$ can be taken as a basis for $\mathbb{R}^{2 g}$, so the subgroup $\mathscr{L}=\Lambda \mathbb{Z}^{2 g} \subset \mathbb{C}^{g}$ generated by these column vectors can be identified with the lattice subgroup $\mathbb{Z}^{2 g} \subset \mathbb{R}^{2 g}$. Topologically the quotient group $\mathbb{C}^{g} / \mathscr{L}$ is thus just the $2 g$-dimensional torus $\mathbb{R}^{2 g} / \mathbb{Z}^{2 g}=(\mathbb{R} / \mathbb{Z})^{2 g}$, a compact manifold homeomorphic to the Cartesian product of $2 g$ circles. On the other hand the quotient group 
$\mathbb{C}^{g} / \mathscr{L}$ also has the structure of a complex manifold, hence of a complex Lie group, the coordinates in $\mathbb{C}^{g}$ providing local coordinates on $\mathbb{C}^{g} / \mathscr{L}$. This complex Lie group is called the Jacobi variety of $M$ and is denoted by $J(M)$; the zero element is the image in $\mathbb{C}^{g} / \mathscr{L}$ of the origin $O \in \mathbb{C}^{g}$. For the special case $g=1$ this construction is quite familiar from the classical theory of elliptic functions. It should be mentioned that although the construction was based on special choices of the paths $\alpha_{j}, \beta_{j}$ and the basis $\omega_{i}$, the resulting complex Lie group is really independent of these choices; however the choice of the basis $\omega_{i}$ so that the period matrix has the special form $\Lambda=(I, \Omega)$, with $\Omega \in \mathfrak{S}_{g}$, really amounts to a somewhat finer structure than just that of a complex Lie group. Nothing further will be made of that point here though, since the effects of changes in the choices of $\alpha_{j}, \beta_{j}, \omega_{i}$ will not be considered at all.

For any point $p \in M$ the vector $\int_{p_{0}}^{p} \boldsymbol{\omega} \in \mathbb{C}^{g}$ depends on the path of integration from $p_{0}$ to $p$; but a change in the choice of this path has the effect of adding a vector $\int_{\gamma} \omega$ for some closed cycle $\gamma$ on $M$, and the set of all these vectors is precisely the lattice subgroup $\mathscr{L}=\Lambda \mathbf{Z}^{2 g} \subset \mathbb{C}^{g}$. It is thus possible to introduce a well-defined holomorphic mapping $w: M \rightarrow J(M)$ by setting

$$
w(p)=\int_{p_{0}}^{p} \omega(\bmod \mathscr{L}) .
$$

The base point $p_{0} \in M$ is mapped to the zero element of the group $J(M)$, and the effect of choosing a different base point $p_{0}^{*} \in M$ is just to replace the mapping $w$ by the translate $w^{*}$ given by $w^{*}(p)=w(p)-w\left(p_{0}^{*}\right)$. Since $J(M)$ is a group, this mapping $w: M \rightarrow J(M)$ has a natural extension to a homomorphism from the free Abelian group generated by the points of $M$, called the group of divisors on $M$, to $J(M)$; a divisor is really just a formal sum $\grave{D}=\sum_{p \in M} \nu_{p} p$ for some integers $\nu_{p} \in \mathbb{Z}$, only finitely many of which are nonzero, and the extension of the mapping $w$ is that given by setting $w(\delta)=$ $\sum_{p \in M} \nu_{p} w(p)$. To any meromorphic function $f$ not identically equal to zero on $M$ there corresponds its divisor $\mathfrak{D}(f)=\sum_{p \in M} \nu_{p}(f) p$, where $\nu_{p}(f)$ is the order of the function $f$ at the point $p: \nu_{p}(f)=n$ if $f$ has a zero of order $n$ at $p$, $\nu_{p}(f)=-n$ if $f$ has a pole of order $n$ at $p$, and $\nu_{p}(f)=0$ otherwise. It is rather easy to see that if $\mathfrak{D}=\Sigma_{p \in M} \nu_{p} p$ is the divisor of a meromorphic function on $M$ then degree $\delta=\sum_{p \in M} \nu_{p}=0$ and $w(\delta)=\Sigma_{p \in M} \nu_{p} w(p)=0$; Abel's theorem asserts that, conversely, any divisor $\delta$ for which degree $\delta=0$ and $w(\delta)=0$ is the divisor of a meromorphic function on $M$. This is a very useful result, one of the basic tools in the study of Riemann surfaces, and the impetus behind the introduction of the Jacobi variety; it indicates that the torus $J(M)$ must contain a great deal of information about the Riemann surface $M$, and thus raises the problem of how to get at that information, a very appealing problem analytically since there is a quite well-developed function theory on complex tori generalizing the classical theory of elliptic functions.

There is a special family of holomorphic subvarieties of a Jacobi variety $J(M)$, the subvarieties of special positive divisors, that reflect in their structure and interrelations many of the finer properties of the Riemann surface $M$. The simplest is just the image of the Riemann surface $M$ in its Jacobi variety, the 
subvariety $W_{1}=w(M)$. It follows quite easily from Abel's theorem that the holomorphic mapping $w: M \rightarrow W_{1}$ is one-one. Indeed if that were not the case there would be two distinct points $p_{1}, p_{2}$ of $M$ such that $w\left(p_{1}\right)=w\left(p_{2}\right)$; then $w\left(p_{1}-p_{2}\right)=0$, so by Abel's theorem there would be a meromorphic function $f$ on $M$ with divisor $\mathfrak{b}(f)=p_{1}-p_{2}$. As is quite familiar, any such function can be viewed as a holomorphic mapping $f: M \rightarrow \mathbb{P}^{1}$ from $M$ to the Riemann sphere. Now for any complex constant $c$ the meromorphic function $f-c$ would also have a simple pole at $p_{2}$, hence would have a divisor of the form $\delta(f-c)=p_{1}^{c}-p_{2}$ for some point $p_{1}^{c} \in M$, which point would of course then be the unique point of $M$ at which the function $f$ took the value $c$; but that means that $f: M \rightarrow \mathbb{P}^{1}$ would be a one-one mapping, hence a homeomorphism, contradicting the initial assumption that $M$ has genus $g>0$. Thus $w: M \rightarrow W_{1}$ is one-one, so can be viewed as a holomorphic injection of $M$ into its Jacobi variety $J(M)$. Using another of the basic tools in the study of Riemann surfaces, the Riemann-Roch theorem, it is relatively easy to show that the mapping $w: M \rightarrow W_{1}$ is nonsingular at each point, hence is biholomorphic; thus $w: M \rightarrow W_{1}$ is actually an imbedding of $M$ as a nonsingular curve in its Jacobi variety $J(M)$. In a sense, of course, knowing $W_{1}$ amounts to knowing all about the Riemann surface $M$, since $W_{1}$ and $M$ are biholomorphic; but the problem is how to determine $W_{1}$ directly from the torus $J(M)$ or its period matrix $\Lambda$. It is worth noting that the curve $W_{1} \subseteq J(M)$ depends on a choice of base point $p_{0}$, and that the choice of a different base point $p_{0}^{*}$ has the effect of replacing $W_{1}$ by the translate $W_{1}-w\left(p_{0}^{*}\right)$; thus what is independent of the choice of base point is the collection of curves $\left\{W_{1}-w_{1}: w_{1} \in W_{1}\right\}$ or their union $W_{1}-W_{1}=\left\{w_{1}-w_{2}: w_{j} \in W_{1}\right\}$. The latter subvariety is a very interesting intrinsically defined two-dimensional subvariety of $J(M)$ and plays a prominent role in the subsequent discussion.

The subvariety $W_{1}$ is sometimes called the subvariety of positive divisors of degree 1 , a divisor $\delta=\sum_{p \in M} \nu_{p} p$ being considered positive if $\nu_{p} \geqslant 0$ for all $p$ so that a positive divisor of degree 1 is really one of the form $\delta=1 \cdot p$, and $W_{1}=\{w(1 \cdot p): p \in M\}$. There is correspondingly a subvariety $W_{r}=$ $\left\{w\left(1 \cdot p_{1}+\cdots+1 \cdot p_{r}\right): p_{j} \in M\right\}$, the subvariety of positive divisors of degree $r$, for any integer $r>0$, and it is sometimes convenient to set $W_{0}=0$, the zero element of the group $J(M)$. These are all irreducible holomorphic subvarieties of $J(M)$ with $W_{0} \subseteq W_{1} \subseteq W_{2} \subseteq \cdots$, and $\operatorname{dim} W_{r}=r$ whenever $1 \leqslant r \leqslant g$, while $W_{g}=W_{g+1}=\cdots=J(M)$. It is quite clear that all of these subvarieties can be constructed directly from $W_{1}$, since

$$
W_{r}=W_{1}+\cdots+W_{1}=\left\{w_{1}+\cdots+w_{r}: w_{j} \in W_{1}\right\}
$$

more generally they nicely reflect the group structure of $J(M)$ in the sense that

$$
W_{r+s}=W_{r}+W_{s}=\left\{w_{1}+w_{2}: w_{1} \in W_{r}, w_{2} \in W_{s}\right\}
$$

for any indices $0 \leqslant r, s$. These subvarieties too depend on the choice of base point $p_{0}$, and the choice of a different base point $p_{0}^{*}$ has the effect of replacing $W_{r}$ by the translate $W_{r}-r w\left(p_{0}^{*}\right)$. 
Much more interesting though is the subvariety $W_{r}^{\nu}$ of positive divisors of degree $r$ and dimension $\nu$, defined by

$$
\begin{aligned}
& W_{r}^{\nu}=\left\{w(\mathfrak{D}): \mathfrak{d}=1 \cdot p_{1}+\cdots+1 \cdot p_{r}\right. \text { and the vector space } \\
& \text { of all meromorphic functions } f \text { on } M \text { with } \\
& \qquad \delta(f)+\delta \text { positive has dimension }>\nu\} .
\end{aligned}
$$

If $\downarrow=1 \cdot p_{1}+\cdots+1 \cdot p_{r}$ the condition that $\delta(f)+\delta$ be positive is just that the meromorphic function $f$ have as singularities at most simple poles at the points $p_{j}$ if they are all distinct or multiple poles corresponding to coincidences among them. For any such divisor there is always at least the one-dimensional vector space of constant functions $f$ for which $\delta(f)+\delta$ is positive, so $W_{r}^{0}=W_{r}$; of course, $W_{r}^{0} \supseteq W_{r}^{1} \supseteq W_{r}^{2} \supseteq \cdots$. These are all holomorphic subvarieties of $J(M)$, but of rather varying dimensions and structures reflecting various properties of the Riemann surface $M$; that is easily illustrated, and the possible interest of these subvarieties indicated, by an examination of some of the simplest cases. First, if $w(1 \cdot p) \in W_{1}^{1}$ there are at least two linearly independent meromorphic functions having as singularities at most a simple pole at $p$; one of these functions must be nonconstant, but, as already noted, is then a homeomorphism $f: M \rightarrow \mathbb{P}^{1}$, contradicting the assumption that $g>0$. Thus $W_{1}^{1}=\varnothing$. Next if $w\left(1 \cdot p_{1}+1 \cdot p_{2}\right) \in W_{2}^{1}$ there are at least two linearly independent meromorphic functions having as singularities at most simple poles at the points $p_{1}, p_{2}$ if they are distinct or a double pole at the point $p_{1}=p_{2}$ if they coincide; one of these functions must be nonconstant and cannot have just a single simple pole since $W_{1}^{1}=\varnothing$, so it must be of order two and, consequently, when viewed as a holomorphic mapping $f: M \rightarrow \mathbb{P}^{1}$, exhibits $M$ as a two-sheeted branched covering of the Riemann sphere. The points $q_{1}, q_{2}$ of $M$ having the same image $f\left(q_{1}\right)=f\left(q_{2}\right)=c$ are those for which $\delta(f-c)=1 \cdot q_{1}+1 \cdot q_{2}-1 \cdot p_{1}-1 \cdot p_{2}$, so by Abel's theorem are precisely the points for which $w\left(1 \cdot q_{1}+1 \cdot q_{2}\right)=w\left(1 \cdot p_{1}+1 \cdot p_{2}\right)$; thus the distinct points of $W_{2}^{1}$ correspond to distinct representations of $M$ as a two-sheeted branched covering of $\mathbb{P}^{1}$. There are Riemann surfaces of arbitrary genus that can be so represented, namely the Riemann surfaces of the functions $P(z)^{1 / 2}$ for any polynomials $P(z)$; but for $g>2$ not all Riemann surfaces can be so represented, only the special subclass called the hyperelliptic surfaces, and it is known that each of these surfaces has a unique such representation. Thus $W_{2}^{1} \neq \varnothing$ only when $M$ is a hyperelliptic surface and then $W_{2}^{1}$ consists of a single point of $J(M)$. Similarly $W_{3}^{1} \neq \varnothing$ only when $M$ is either hyperelliptic or can be represented as a three-sheeted branched covering of the Riemann sphere, and the points of $W_{3}^{1}$ correspond to the number of representations of $M$ as a two- or three-sheeted cover; it is known that $W_{3}^{1}$ is a translate of $W_{1}$ if $M$ is hyperelliptic, but otherwise is either a finite point set or the empty set.

The various subvarieties $W_{r}^{\nu}$ are interrelated in a variety of ways involving the group structure of $J(M)$, so although there is a rather wide range of possibilities for the configuration of all of them there are also quite definite restrictions. The detailed study of these interrelations has been very effectively 
used by H. H. Martens among others [30], and the investigation of these subvarieties and their properties is still being quite actively pursued, as is evident upon looking at [ 3 or 36] for example. Just to illustrate some of the interrelations, it may be of interest to see how these various subvarieties can all be described in terms of $W_{1}$ and the group structure of $J(M)$. It was already noted that $W_{r}=W_{1}+\cdots+W_{1}$, and it is natural to set $-W_{r}=\{-w$ : $\left.w \in W_{r}\right\}$, so these subvarieties are determined quite directly by $W_{1}$, and from these in turn

$$
W_{r}^{\nu}=\left\{w \in J(M): w-W_{\nu} \subseteq W_{r-\nu}\right\} ;
$$

alternatively it is possible to describe the subvarieties $W_{r}^{\nu}$ inductively on $\nu$ by using the subvariety $W_{1}-W_{1}$ mentioned earlier, since

$$
W_{r}^{\nu}=\left\{w \in J(M): w+W_{1}-W_{1} \subseteq W_{r}^{\nu-1}\right\}
$$

3. For any matrix $\Omega \in \mathscr{S}_{g}$ the extended matrix $\Lambda=(I, \Omega)$ can be used to generate a lattice subgroup $\mathscr{L}=\Lambda \mathbb{Z}^{2 g} \subset \mathbb{C}^{g}$, to which there is associated the compact complex torus $J(\Omega)=\mathbb{C}^{g} / \mathscr{L}$. In studying such tori the Jacobian theta functions have long been basic tools, providing quite explicit representations for the meromorphic functions on $J(\Omega)$ and descriptions of some of the holomorphic subvarieties of $J(\Omega)$. That suggests attempting to describe the subvarieties of special positive divisors $W_{r}^{\nu}$ in the Jacobi varieties of Riemann surfaces in terms of these theta functions, but in such an endeavor two problems must be kept in mind. The first is that whereas the theta functions provide quite simple and explicit descriptions of the subvarieties of codimension one, they generally only provide rather cumbersome and complicated descriptions for subvarieties of lower dimensions; ideally a theory of vectorvalued theta functions should be a more tractable tool for handling lowerdimensional subvarieties of complex tori, but none has as yet been sufficiently explicitly developed. The second is that whereas the theta functions can be introduced for any period matrix $\Omega \in \mathscr{F}_{g}$, only a special subset of the matrices $\Omega \in \mathfrak{S}_{g}$ are the normalized period matrices of compact Riemann surfaces; thus any general expression in terms of the theta functions can only be expected to describe subvarieties of the dimensions and other properties desired for special values of $\Omega$, an observation that has underlaid several approaches to the problem of characterizing those matrices $\Omega \in \mathscr{F}_{g}$ that are the normalized period matrices of compact Riemann surfaces.

The basic theta series associated to a matrix $\Omega \in \mathfrak{G}_{g}$ is

$$
\theta[\nu \mid \tau](w ; \Omega)=\sum_{n} \exp 2 \pi i\left[\frac{1}{2}^{\mathrm{t}}(n+\nu) \Omega(n+\nu)+{ }^{\mathrm{t}}(n+\nu)(w+\tau)\right],
$$

where the summation is extended over all column vectors of integers $n \in \mathbb{Z}^{g}$; this is to be viewed as a series of terms in the variables $w \in \mathbb{C}^{g}$, with additional parameters $\nu, \tau \in \mathbb{C}^{g}$ called the characteristics. Since $\Omega \in \mathscr{S}_{g}$ the imaginary part of $\Omega$ is positive definite, so if $\gamma>0$ is its smallest eigenvalue then the absolute value of the $n$th term of the series (5) is bounded from above by $\exp \left(-\pi \gamma^{\mathrm{t}} n \cdot n+{ }^{\mathrm{t}} n|w|+{ }^{\mathrm{t}} n \alpha+\beta\right)$ for some constants $\alpha, \beta$, therefore the 
series clearly converges locally absolutely uniformly, hence represents an entire function of the $g$ complex variables $w \in \mathbb{C}^{g}$. The parameters $\nu, \tau$ really just describe a translation of the coordinates in the elementary theta function

$$
\theta(w)=\theta[0 \mid 0](w ; \Omega)
$$

as is evident from the identity

$$
\theta[\nu \mid \tau](w ; \Omega)=\theta(w+\Omega \nu+\tau) \exp 2 \pi i\left[\frac{1}{2}{ }^{\mathrm{t}} \nu \Omega \nu+{ }^{\mathrm{t}} \nu(w+\tau)\right]
$$

but are convenient and traditional; the notation adopted here is a slight variant of one of the customary ones, to simplify life for the printer.

The basic property of the theta function is that for any lattice vector $\lambda \in \mathscr{L}$ there is a nowhere-vanishing entire function $\eta(\lambda, w)$ of the $g$ complex variables $w \in \mathbb{C}^{g}$ such that

$$
\theta(w+\lambda)=\eta(\lambda, w) \theta(w) .
$$

The zero locus of the function $\theta$ is thus a holomorphic subvariety of pure dimension $g-1$ in $\mathbb{C}^{g}$ and is invariant under the lattice subgroup $\mathscr{L}$, so it determines a holomorphic subvariety of pure dimension $g-1$ in the torus $J(\Omega)=\mathbb{C}^{g} / \mathscr{L}$; this latter subvariety is called the theta locus and will be denoted by $\Theta$. It is thus a canonically constructed subvariety of the torus $J(\Omega)$, described quite simply and explicitly in terms of the period matrix $\Omega$ alone as the zero locus of the elementary theta series $\theta(w)$.

The significance of the theta locus $\Theta$ for Riemann surfaces was recognized by Riemann in one of the most glorious theorems of the subject. The first part of Riemann's theorem is the assertion that if $\Omega$ is the period matrix of a compact Riemann surface $M$, so that the torus $J(\Omega)$ can be identified with the Jacobi variety $J(M)$, then

$$
W_{g-1}=\Theta+R
$$

for some point $R \in J(M)$; thus the subvariety $W_{g-1}$ of positive divisors of degree $g-1$ is determined quite simply and explicitly by the period matrix $\Omega$ alone, at least up to a translation. The point $R$ is uniquely determined by (9) and can be written down rather explicitly in terms of the Riemann surface $M$, although not in terms just of the torus $J(M)$; it and $W_{g-1}$ do depend on the choice of the base point of $M$. There is even more to Riemann's theorem, though, involving the locus points of order $\nu$ of the elementary theta function $\theta(w)$, the holomorphic subvariety $\Theta^{\nu} \subseteq \Theta$ defined as

$$
\begin{array}{r}
\Theta^{\nu}=\{w \in J(\Omega): \theta \text { and all partial derivatives of } \theta \\
\text { of orders } \leqslant \nu \text { vanish at } w\} / \mathscr{L} .
\end{array}
$$

The second part of the theorem asserts that

$$
W_{g-1}^{\nu}=\Theta^{\nu}+R
$$

thus all the subvarieties $W_{g-1}^{\nu}$ of special positive divisors of dimensions $\nu=0,1,2, \ldots$ are also determined quite simply and explicitly by the period matrix $\Omega$ alone, up to translation. In particular, the theta function $\theta$ vanishes to 
the first order at $\Theta$, hence generates the proper ideal of that subvariety at each of its points, and $W_{g-1}^{1}$ is precisely the singular locus of $W_{g-1}$. Riemann's work can be found in [37], and modern proofs in [28, 31, and 33], among other places. As a consequence of Riemann's theorem any results derived only from a knowledge of the subvarieties $W_{g-1}^{\nu}$ up to translation are really determined by the period matrix $\Omega$ alone. This observation is the basis for most current proofs of Torelli's theorem that any two Riemann surfaces having the same normalized period matrix $\Omega$ are biholomorphic [1, 29, 32, 39]. Although this does mean that the Riemann surface $M$ itself and hence all of the subvarieties $W_{r}^{\nu}$ are to some extent determined by the period matrix $\Omega$ alone, the actual way in which they are determined is rather indirect and too ineffective for some purposes.

4. There is thus some point to examining further the use of theta functions to describe various subvarieties of complex tori, and in this examination the second-order theta functions play a prominent role; they are defined by

$$
\theta_{2}[\nu \mid \tau](w ; \Omega)=\theta[\nu \mid 2 \tau](2 w ; 2 \Omega),
$$

so are really just a simple variant of the standard theta functions. The basic property of the second-order theta functions is that whenever $\nu$ is a half-integral vector, so that $2 \nu \in \mathbb{Z}^{g}$, then, for any lattice vector $\lambda \in \mathscr{L}$,

$$
\theta_{2}[\nu \mid 0](w+\lambda ; \Omega)=\eta(\lambda, w)^{2} \theta_{2}[\nu \mid 0](w ; \Omega),
$$

where $\eta(\lambda, w)$ is the nowhere-vanishing entire function of $w$ that had earlier appeared in (8). As long as none of the vectors $\nu$ differ by an integral vector, the corresponding second-order theta functions are linearly independent, as is evident from the Fourier expansion (5), while $\theta_{2}[\nu+m \mid 0](w ; \Omega)=$ $\theta_{2}[\nu \mid 0](w ; \Omega)$ for any integral vector $m$, as is also evident from (5); thus as $\nu$ varies over the group $(\mathbb{Z} / 2 \mathbb{Z})^{g}$, or equivalently as $\nu$ varies over the set of rational vectors with entries either 0 or $1 / 2$, the corresponding second-order theta functions range over $2^{g}$ linearly independent entire functions of $w$ satisfying the functional equation (12)-indeed they form a basis for the complex vector space of entire functions satisfying (12). For notational convenience set

$$
\theta_{2}(w)=\left\{\theta_{2}[\nu \mid 0](w ; \Omega): \nu \in(\mathbb{Z} / 2 \mathbb{Z})^{g}\right\},
$$

viewed as a column of length $2^{g}$ consisting of entire functions of $w$ or, alternatively, as a holomorphic mapping $\theta_{2}: \mathbb{C}^{g} \rightarrow \mathbb{C}^{G}$ where $G=2^{g}$. The square of the elementary theta function $\theta(w)$ satisfies (12), so it can be written $\theta(w)^{2}={ }^{t} c \cdot \theta_{2}(w)$ for some vector $c \in \mathbb{C}^{g}$; both more general and more precise is the relation between the elementary and second-order theta functions given by Weierstrass's formula

$$
\theta(w+\alpha) \theta(w+\beta)={ }^{\mathrm{t}} \theta_{2}\left(\frac{\alpha-\beta}{2}\right) \cdot \theta_{2}\left(w+\frac{\alpha+\beta}{2}\right)
$$

or the dual form corresponding to the special case $\beta=-\alpha$,

$$
{ }^{\mathrm{t}} \theta_{2}(\alpha) \cdot \theta_{2}(w)=\theta(w+\alpha) \theta(w-\alpha) .
$$


There is now a very natural way in which the second-order theta functions can be used to describe subvarieties of the complex tori $J(\Omega)$. The vector $\theta_{2}(w)$ is never the zero vector; indeed if $\theta_{2}(\alpha)=0$ for some point $\alpha$ then it follows from $\left(14^{\prime}\right)$ that $\theta(w+\alpha) \theta(w-\alpha)=0$ for all $w$, an evident impossibility. Thus this vector can be viewed as describing a point $\left[\theta_{2}(w)\right]$ in the projective space $\mathbb{P}^{G-1}$ of dimension $G-1=2^{g}-1$. The functional equation (12) shows that $\theta_{2}(w)$ and $\theta_{2}(w+\lambda)$ describe the same point in $\mathbb{P}^{G-1}$ for any lattice vector $\lambda \in \mathscr{L}$, so there results a well-defined holomorphic mapping

$$
\left[\theta_{2}\right]: J(\Omega) \rightarrow \mathbb{P}^{G-1} .
$$

Then for any subvariety $[S] \subseteq \mathbb{P}^{G-1}$ the subset $\left\{w \in J(\Omega):\left[\theta_{2}(w)\right] \in[S]\right\}$ is a well-defined subvariety of $J(\Omega)$. The problem of course is that of determining subvarieties $[S] \subseteq \mathbb{P}^{G-1}$ for which the corresponding subvarieties of $J(\Omega)$ are of an intrinsic interest, such as being related to the subvarieties $W_{r}^{\nu}$ of special positive divisors when $\Omega$ is the period matrix of a Riemann surface.

Before turning to a discussion of that problem though, a few further remarks about the mapping (15) and its geometrical properties should be added here. The image of this mapping is an algebraic subvariety $K(\Omega) \subseteq \mathbb{P}^{G-1}$ canonically associated to the period matrix $\Omega$, called the Wirtinger variety for $\Omega$; in the special case $g=2$ it is just Kummer's quartic surface [24], a much studied algebraic surface in $\mathbb{P}^{3}$ of which the Wirtinger varieties are thus natural generalizations. If the theta locus $\Theta$ is assumed to be irreducible, a rather reasonable nondegeneracy condition that by (9) is automatically satisfied whenever $\Omega$ is the normalized period matrix of a Riemann surface, then, as Wirtinger demonstrated in [44], the mapping $\left[\theta_{2}\right]: J(\Omega) \rightarrow K(\Omega)$ is a twosheeted branched covering with double points at the $2^{2 g}$ half-periods in $J(\Omega)$, but otherwise nonsingular; that is the best that could be expected, since the second-order theta functions $\theta_{2}[\nu \mid 0](w ; \Omega)$ for halfintegral vectors $\nu$ are all even functions of $w$ and hence $\theta_{2}(-w)=\theta_{2}(w)$. The Wirtinger variety $K(\Omega)$ can thus be identified with the quotient $J(\Omega) / E$ of the torus $J(\Omega)$ under the holomorphic involution $E: J(\Omega) \rightarrow J(\Omega)$ induced by the mapping $w \rightarrow-w$; the half-periods in $J(\Omega)$ are precisely the fixed points of $E$. The subvarieties of $J(\Omega)$ to be considered next are just the inverse images under (15) of the intersections $K(\Omega) \cap[S] \subseteq \mathbb{P}^{G-1}$; it should be noted that all such subvarieties are invariant under the involution $E$.

For some purposes it is also of interest to consider the mapping $\theta_{2}^{*}$ : $\mathbb{C}^{g} \rightarrow \mathbb{C}^{G}$ defined by $\theta_{2}^{*}(w)=\theta_{2}(w / 2)$; the functional equation for the mapping $\theta_{2}^{*}$ under translation by a period vector reflects the transformational properties of the second-order theta functions under translation by a half-period and is a bit more complicated than (12), but well established [23, 34]. For any lattice vector $\lambda \in \mathscr{L}$ there is a nonsingular $G \times G$ matrix $\chi(\lambda)$, with entries either $0,+1$, or -1 , such that

$$
\theta_{2}^{*}(w+\lambda)=\eta(\lambda, w)^{1 / 2} \chi(\lambda) \theta_{2}^{*}(w) ;
$$

then $\chi\left(\lambda_{1}+\lambda_{2}\right)= \pm \chi\left(\lambda_{1}\right) \chi\left(\lambda_{2}\right)$ for any lattice vectors $\lambda_{1}, \lambda_{2} \in \mathscr{L}$, while branches of the square roots can be so chosen that $\chi(2 \lambda)=I$ for any lattice 
vector $\lambda \in \mathscr{L}$. If $\chi^{*}(\lambda): \mathbb{P}^{G-1} \rightarrow \mathbb{P}^{G-1}$ is the projective transformation represented by the matrix $\chi(\lambda)$ then the mapping $\lambda \rightarrow \chi^{*}(\lambda)$ is a homomorphism from $\mathscr{L}$ into the group of projective transformations on $\mathbb{P}^{G-1}$ with a kernel including $2 \mathscr{L}$, and (16) implies that $\left[\theta_{2}^{*}(w+\lambda)\right]=\chi^{*}(\lambda)\left[\theta_{2}^{*}(w)\right]$; the image $\mathscr{G}=\left\{\chi^{*}(\lambda): \lambda \in \mathscr{L}\right\}$ is therefore a finite group of projective transformations mapping the Wirtinger variety $K(\Omega) \subseteq \mathbb{P}^{G-1}$ to itself, and with the nondegeneracy condition that $\Theta$ be irreducible, as before, the induced mapping $\left[\theta_{2}^{*}\right]$ : $J(\Omega) / E \rightarrow K(\Omega) / \mathscr{G}$ is a one-to-one holomorphic mapping.

5. To consider now some explicit examples of this method of describing subvarieties of $J(\Omega)$, to any fixed set of indices $1 \leqslant j_{1}, \ldots, j_{\lambda} \leqslant g$ associate the vector

$$
\partial_{j_{1} \cdots j_{\lambda}} \theta_{2}(0)=\left.\frac{\partial^{\lambda} \theta_{2}(w)}{\partial w_{j_{1}} \cdots \partial w_{j_{\lambda}}}\right|_{w=0} \in \mathbb{C}^{G} ;
$$

this vector is of course zero if $\lambda$ is odd, since the second-order theta functions involved are even functions, and is symmetric in the induces $j_{1}, \ldots, j_{\lambda}$. In terms of these vectors let

$$
S_{\nu}=\operatorname{span}\left\{\theta_{2}(0), \partial_{j_{1} j_{2}} \theta_{2}(0), \ldots, \partial_{j_{1} \cdots j_{2 v}} \theta_{2}(0)\right\} \subseteq \mathbb{C}^{G},
$$

where by this is meant the linear subspace of $\mathbb{C}^{G}$ spanned by all the vectors listed for all choices of indices $j_{i}$, and let $\left[S_{\nu}\right]$ denote the image of this linear subspace in the projective space $\mathbb{P}^{G-1}$. Dually let

$$
S_{\nu}^{\perp}=\left\{s \in \mathbb{C}^{G}:{ }^{\mathrm{t}} s \cdot t=0 \text { for all } t \in S_{\nu}\right\},
$$

where ${ }^{\mathrm{t}} s \cdot t=\sum s_{j} t_{j}$ is the standard inner product in $\mathbb{C}^{G}$, and let $\left[S_{\nu}^{\perp}\right]$ denote the image of this linear subspace in the projective space $\mathbb{P}^{G-1}$. The first example is a very simple consequence of Weierstrass's formula (14'); the special case $\nu=0$ is indeed both familiar and trivial, the special case $\nu=1$ was used in [2], and there must have been many other earlier occurrences.

THEOREM 1. $\left[\theta_{2}\right]^{-1}\left(K(\Omega) \cap\left[S_{\nu}^{\perp}\right]\right)=\Theta^{\nu}$.

\section{Proof. Setting}

$$
\Phi^{\nu}=\left\{w \in J(\Omega):{ }^{\mathrm{t}} s \cdot \theta_{2}(w)=0 \text { for all } s \in S_{\nu}\right\},
$$

the assertion of the theorem is just that $\Phi^{\nu}=\Theta^{\nu}$, the locus of points of order $\nu$ of the elementary theta function. This result will be demonstrated by induction on $\nu$. For the initial case $\nu=0$ note from $\left(14^{\prime}\right)$ that

$$
\Phi^{0}=\left\{w \in J(\Omega): 0={ }^{\mathrm{t}} \theta_{2}(0) \cdot \theta_{2}(w)=\theta(w)^{2}\right\},
$$

hence that $\Phi^{0}=\Theta$ as desired. Assuming then that the result holds for $\nu-1 \geqslant 0$, it follows that

$$
\begin{aligned}
& \Phi^{\nu}=\{w \in J(\Omega): 0=\theta(w)=\partial_{j_{1}} \theta(w)=\cdots=\partial_{j_{1} \cdots j_{\nu-1}} \theta(w) \\
&\left.={ }^{t} \partial_{j_{1} \cdots j_{2 v}} \theta_{2}(0) \cdot \theta_{2}(w) \text { for all indices } j_{i}\right\} .
\end{aligned}
$$


Setting $J=\left(j_{1} \cdots j_{2 v}\right)$, note that by applying the product rule for differentiation to $\left(14^{\prime}\right)$ it follows that

$$
0={ }^{\mathrm{t}} \partial_{J} \theta_{2}(0) \cdot \theta_{2}(w)=\sum_{J=J^{\prime} \cup J^{\prime \prime}} \partial_{J^{\prime}} \theta(w) \partial_{J^{\prime \prime}} \theta(w)
$$

where the summation is extended over all ways of writing $J$ as a union of subsets of indices $J^{\prime}, J^{\prime \prime}$; the induction hypothesis shows that the only nontrivial terms in this summation arise when both $J^{\prime}$ and $J^{\prime \prime}$ consist of $\nu$ of the indices in $J$. It is a fairly straightforward algebraic observation, possibly most readily seen by another induction on $\nu$, that this identity implies that $\partial_{j_{1} \ldots j_{\nu}} \theta(w)=0$ for all indices $j_{i}$, thereby concluding the proof.

The preceding result shows that for any fixed $\nu$ the subvariety $\Theta^{\nu} \subseteq J(\Omega)$ can be described as the set of common zeros of a particular set of second-order theta functions, namely the functions ${ }^{\mathrm{t}} s \cdot \theta_{2}(w)$, where $s$ ranges either over all of $S_{\nu}$ or just over a basis of $S_{\nu}$. In particular,

$$
\Theta=\left\{w \in J(\Omega):{ }^{\mathrm{t}} \theta_{2}(0) \cdot \theta_{2}(w)=0\right\},
$$

while the singular locus of $\Theta$ is

$$
\Theta^{1}=\left\{w \in \Theta:{ }^{t} \partial_{j_{1} j_{2}} \theta_{2}(0) \cdot \theta_{2}(w)=0 \text { for } 1 \leqslant j_{1} \leqslant j_{2} \leqslant g\right\} ;
$$

since there are $\left(g_{2}^{+1}\right) \geqslant g$ of the latter equations, it would be expected that $\Theta^{1}=\varnothing$ in general, that is, that the locus $\Theta$ is nonsingular, as in the case.

This immediately suggests other explicit examples of this method of describing subvarieties of $J(\Omega)$; while the descriptions are quite easy, it is generally rather difficult to say much of anything at all about the subvarieties so described. Only two families of such subvarieties will be considered here. Firstly, and most obviously, for any integer $\nu \geqslant 0$ introduce the subvariety

$$
X_{\nu}=\left[\theta_{2}\right]^{-1}\left(K(\Omega) \cap\left[S_{\nu}\right]\right) \subseteq J(\Omega),
$$

which can also be described as the set of common zeros of a particular set of second-order theta functions in the form

$$
X_{\nu}=\left\{w \in J(\Omega):{ }^{t} s \cdot \theta_{2}(w)=0 \quad \text { for all } s \in S_{\nu}^{\perp}\right\} .
$$

Secondly, and somewhat more subtly, in Theorem 1 it is quite conceivable that the intersection $K(\Omega) \cap\left[S_{\nu}^{\perp}\right]$ only spans a proper subspace of $\left[S_{\nu}^{\perp}\right]$, or, equivalently, that the intersection subvariety can also be described as $K(\Omega) \cap$ [T] for a proper linear subspace $T \subset S_{\nu}^{\perp}$; therefore let

$$
\begin{aligned}
T_{\nu}^{\perp}= & \text { smallest linear subspace } T \subseteq S_{\nu}^{\perp} \subseteq \mathbb{C}^{G} \\
& \text { for which } K(\Omega) \cap[T]=K(\Omega) \cap\left[S_{\nu}^{\perp}\right],
\end{aligned}
$$

or, equivalently, let $T_{\nu}^{\perp}$ be the linear subspace of $\mathbb{C}^{G}$ spanned by all representatives of all points in $K(\Omega) \cap\left[S_{\nu}^{\perp}\right] \subseteq \mathbb{P}^{G-1}$, and dually set

$$
T_{\nu}=\left\{t \in \mathbb{C}^{G}:{ }^{t} t \cdot s=0 \text { for all } s \cap T_{\nu}^{\perp}\right\} .
$$

In view of Theorem 1 the latter subspace can also be described as

$$
T_{\nu}=\left\{t \in \mathbb{C}^{G}:{ }^{t} t \cdot \theta_{2}(w)=0 \text { for all } w \in \Theta^{\nu}\right\} .
$$


Then introduce the subvariety

$$
Y_{\nu}=\left[\theta_{2}^{-1}\right]\left(K(\Omega) \cap\left[T_{\nu}\right]\right),
$$

which is again the set of common zeros of the appropriate set of second-order theta functions.

Since $T_{\nu}^{\perp} \subseteq S_{\nu}^{\perp}$ by definition, it must be the case dually that $T_{\nu} \supseteq S_{\nu}$ and, consequently,

$$
X_{\nu} \subseteq Y_{\nu}
$$

for every index $\nu$; as will eventually be demonstrated, this is sometimes, but not always, a strict inclusion. Although more complicated to define, the subvarieties $Y_{\nu}$ are rather easier to handle than the subvarieties $X_{\nu}$; but on the other hand the subvarieties $X_{v}$ are evidently of a more independent interest. To describe the subvarieties $Y_{\nu}$ in another manner, it is convenient to use a notion and notation introduced by $\mathrm{H}$. H. Martens: for any subsets $A, B$ of the torus $J(\Omega)$ set

$$
A \ominus B=\{w \in J(\Omega): w+B \subseteq A\} .
$$

Note that if $A, B$ are holomorphic subvarieties of the Abelian variety $J(\Omega)$ then $A \ominus B=\bigcap_{t \in B}(A-t)$ is also a holomorphic subvariety. It is perhaps worth pointing out here that $W_{r} \ominus W_{s}=W_{r-s}$ whenever $r \geqslant s$, complementing the earlier observation that $W_{r}+W_{s}=W_{r+s}$. Now setting $\Theta^{\nu}=\Theta_{1}^{\nu} \cup \Theta_{2}^{\nu} \cup \cdots$, where $\Theta_{j}^{\nu}$ are the irreducible components of the holomorphic subvariety $\Theta^{\nu} \subseteq J(\Omega)$, the following holds.

THEOREM 2. $Y_{\nu}=\bigcap_{j}\left[\left(\Theta \ominus \Theta_{j}^{\nu}\right) \cup\left(\Theta \ominus-\Theta_{j}^{\nu}\right)\right]$.

Proof. Upon using (22) and (23) together with Weierstrass's formula (14') it follows readily that

$$
Y_{\nu}=\left\{y \in J(\Omega): 0={ }^{\mathrm{t}} \theta_{2}(y) \cdot \theta_{2}(w)=\theta(w+y) \theta(w-y) \quad \text { for all } w \in \Theta^{\nu}\right\},
$$

which can be rewritten geometrically as

$$
Y_{\nu}=\left\{y \in J(\Omega): \Theta^{\nu} \subseteq(\Theta-y) \cup(\Theta+y)\right\} .
$$

Now $\Theta^{\nu} \subseteq(\Theta-y) \cup(\Theta+y)$ precisely when either $\Theta_{j}^{\nu} \subseteq \Theta-y$ or $\Theta_{j}^{\nu} \subseteq \Theta$ $+y$ for each irreducible component $\Theta_{j}^{\nu}$ of $\Theta^{\nu}$, or, equivalently, precisely when $y \in\left(\Theta \ominus \Theta_{j}^{\nu}\right) \cup\left(\Theta \Theta-\Theta_{j}^{\nu}\right)$ for each $j$ since $-\Theta=\Theta$, and that suffices to conclude the proof.

Only for the special case $\nu=0$ can much more very easily be said in general about these subvarieties. Firstly, since $\left[S_{0}\right]$ is just the single point $\left[\theta_{2}(0)\right]$ it follows from the general results of Wirtinger discussed in $\$ 4$ that $X_{0}=0$, at least when the theta locus is assumed irreducible. Under the same assumption it follows from Theorem 2 that $Y_{0}=\Theta \ominus \Theta=0$, hence that $X_{0}=Y_{0}$. Actually, recalling (22), the space $T_{0}$ consists of those vectors $t \in \mathbb{C}^{G}$ such that the second-order theta function ${ }^{\mathrm{t}} t \cdot \theta_{2}(w)$ vanishes on $\Theta$, hence under the same assumption as before such that ${ }^{\mathrm{t}} t \cdot \theta_{2}(w)=\theta(w) h(w)$ for some entire function $h(w)$; but this function $h(w)$ must then satisfy the same functional equation as $\theta(w)$, so it must be a constant multiple of $\theta(w)$, hence $t$ must be unique up to 
an arbitrary scalar multiple. That means $T_{0}$ is one-dimensional, hence $S_{0}=T_{0}$ as well.

6. If $\Omega$ is the normalized period matrix of a compact Riemann surface then something further can be said, and the situation becomes rather more interesting. First the vectors $\theta_{2}(0)$ and $\partial_{j_{1} j_{2}} \theta_{2}(0)$ for $1 \leqslant j_{1} \leqslant j_{2} \leqslant g$ are necessarily linearly independent, a nondegeneracy condition that holds for a general matrix $\Omega \in \mathfrak{S}_{g}$, but not for all matrices $\Omega \in \mathfrak{S}_{g}$; that was observed by Andreotti and Mayer [2], and a correct proof can be found in [9]. Thus

$$
\operatorname{dim} K(\Omega)+\operatorname{dim}\left[S_{1}^{\perp}\right]-\operatorname{dim} \mathbb{P}^{G-1}=g-1-\left(\begin{array}{c}
g+1 \\
2
\end{array}\right)<0 \quad \text { for all } g,
$$

so it would be expected that the subvarieties $K(\Omega)$ and $\left[S_{1}^{\perp}\right]$ of $\mathbb{P}^{G-1}$ would be disjoint and, hence, by Theorem 1 , that $\Theta^{1}=\varnothing$. On the other hand, it is well known that $\operatorname{dim} \Theta^{1}=g-3$ when $M$ is a hyperelliptic Riemann surface, while $\operatorname{dim} \Theta^{1}=g-4$ otherwise, so, in particular, $\Theta^{1} \neq \varnothing$ whenever $g>4$. That means the subvarieties $K(\Omega)$ and $\left[S_{1}^{\perp}\right]$ of $\mathbb{P}^{G-1}$ must be situated in rather special positions with respect to one another whenever $g \geqslant 4$, since their intersection has a larger dimension than that of the intersection of two generically situated subvarieties of $\mathbb{P}^{G-1}$ of the given dimensions. When viewed as a condition on the matrix $\Omega$ this goes quite far towards determining precisely which matrices $\Omega \in \mathscr{S}_{g}$ are the normalized period matrices of compact Riemann surfaces of genus $g$. Indeed, in their fundamental paper [2] Andreotti and Mayer showed that this condition describes a holomorphic subvariety in $\mathfrak{S}_{g}$, and that an irreducible component of this subvariety is the closure of the set of period matrices of compact Riemann surfaces of genus $g$; but it was demonstrated by Beauville in [5] that there are other components.

There are similar, but rather more complicated, results for the space $S_{1}$. In this case

$$
\operatorname{dim} K(\Omega)+\operatorname{dim}\left[S_{1}\right]-\operatorname{dim} \mathbb{P}^{G-1}=\left(\begin{array}{c}
g+2 \\
2
\end{array}\right)-2^{g}<0 \quad \text { for } g \geqslant 4,
$$

so it would be expected that the subvarieties $K(\Omega)$ and $\left[S_{1}\right]$ of $\mathbb{P}^{G-1}$ would be disjoint for $g \geqslant 4$, or, equivalently, in view of definition (19) that $X_{1}=\varnothing$ for $g \geqslant 4$. On the other hand, it follows from results of Fay that $X_{1} \neq \varnothing$ in the Jacobi variety of any compact Riemann surface, so $K(\Omega)$ and $\left[S_{1}\right]$ must also be situated in rather special positions with respect to one another whenever $g \geqslant 4$. What this means as a condition on the matrix $\Omega$, in particular whether it is equivalent to the condition of Andreotti and Mayer, or whether it goes as far towards characterizing the period matrices of Riemann surfaces, has not yet been fully explored. Of course the statement of this condition is not very complete, since to be analogous to the condition of Andreotti and Mayer it should not merely be asserted that $X_{1} \neq \varnothing$ but at least that $X_{1}$ has a specified dimension; as will later be seen there are even stronger forms of this condition that do at least go as far towards characterizing the period matrices of Riemann surfaces as the Andreotti-Mayer condition. 
The further discussion here of the subvariety $X_{1}$ rests on a beautiful result of J. D. Fay, a simple consequence of his trisecant formula as discussed in [13 and 14]; it is the assertion that for any points $p_{1}, p_{2} \in M$ there are complex constants $q, r$ for which

$$
\theta_{2}\left(w\left(p_{1}\right)-w\left(p_{2}\right)\right)=r \cdot \theta_{2}(0)+\frac{1}{2} q^{2} \sum_{j k} w_{j}^{\prime}\left(p_{1}\right) w_{k}^{\prime}\left(p_{2}\right) \cdot \partial_{j k} \theta_{2}(0) .
$$

Note that $w\left(p_{1}\right)-w\left(p_{2}\right)=\int_{p_{2}}^{p_{1}} \omega$ depends on a choice of path from $p_{2}$ to $p_{1}$; the choice of another path has the effect of multiplying the left-hand side of (26) and, hence, the constants $q, r$ by suitable complex constants. Having chosen a path, and having also chosen some local coordinate systems near $p_{1}$ and $p_{2}$ on $M$ in terms of which the differentiations on the right-hand side of (26) are to be carried out, the constants $r=r\left(p_{1}, p_{2}\right)$ and $q=q\left(p_{1}, p_{2}\right)$ are locally well-defined holomorphic functions of $p_{1}$ and $p_{2}$; for some purposes it is convenient to consider these functions as defined on the universal covering surface of $M$, where they are well-defined single-valued holomorphic functions as described in detail in [23]. At any rate it follows immediately from (26) that

$$
W_{1}-W_{1} \subseteq X_{1} \text {, }
$$

and hence that $\operatorname{dim} X_{1} \geqslant 2$ in the Jacobi variety of any compact Riemann surface.

For the Jacobi variety of a hyperelliptic Riemann surface of any genus, the situation is particularly simple. It is no doubt familiar, and is discussed in [20], among other places, that in this case there is a point $E \in J(M)$ such that, $-W_{1}=W_{1}-E$, and, hence, $W_{1}-W_{1}=W_{2}-E$. Combining this with (10) and (4), it follows that

$$
\Theta^{1}=W_{g-1}^{1}-R=\left[W_{g-1} \ominus\left(W_{2}-E\right)\right]-R=W_{g-3}+E-R
$$

using this, and noting that $\Theta^{1}$ is irreducible and $-\Theta^{1}=\Theta^{1}$, it further follows from Theorem 2 that

$$
Y_{1}=\Theta \ominus \Theta^{1}=\left(W_{g-1}-R\right) \ominus\left(W_{g-3}+E-R\right)=W_{2}-E=W_{1}-W_{1} .
$$

Consequently,

$$
X_{1}=Y_{1}=W_{1}-W_{1} \subseteq J(M) \quad \text { when } M \text { is hyperelliptic. }
$$

From this at least the following can readily be deduced in general.

TheOREM 3. $\operatorname{dim} X_{1}=2$ in the Jacobi variety of a general Riemann surface.

Proof. Denoting the vector of second-order theta functions (13) temporarily by $\theta_{2}(w ; \Omega)$ to emphasize that it is a holomorphic function of both $w \in \mathbb{C}^{g}$ and $\Omega \in \mathfrak{G}_{g}$, introduce the holomorphic subvariety

$$
\begin{aligned}
\mathscr{X}_{1}=\{(w, \Omega) & \in \mathbb{C}^{g} \times \mathscr{\mathcal { B }}_{g}: \\
& \left.\operatorname{rank}\left[\theta_{2}(w ; \Omega), \theta_{2}(0 ; \Omega), \partial_{j_{\nu_{2}}} \theta_{2}(0 ; \Omega)\right] \leqslant 1+\left(\begin{array}{c}
g+1 \\
2
\end{array}\right)\right\}
\end{aligned}
$$


and the natural projection $\pi: \mathscr{X}_{1} \rightarrow \mathscr{S}_{g}$. Whenever $\Omega \in \mathscr{S}_{g}$ is the period matrix of a compact Riemann surface then

$$
\operatorname{rank}\left[\theta_{2}(0 ; \Omega), \partial_{j_{1} j_{2}} \theta_{2}(0 ; \Omega)\right]=1+\left(\begin{array}{c}
g+1 \\
2
\end{array}\right)
$$

as already noted, so $X_{1}=\pi^{-1}(\Omega)$ when this subvariety of $\mathbb{C}^{g}$ is viewed as a subvariety of $J(\Omega)$. The desired assertion follows quite easily upon using a few general results form complex analysis in several variables, results that can be found in [15], among other places, together with some results on Teichmüller spaces in the survey [6]. First the subset $\mathscr{X}_{1}^{*} \subseteq \mathscr{X}_{1}$ consisting of all those points $(w, \Omega) \in \mathscr{X}_{1}$ at which the subvariety $\pi^{-1}(\pi(w, \Omega))$ has dimension $>2$ is a holomorphic subvariety of $\mathscr{X}_{1}$. Next since $\pi^{-1}(\Omega) \cap \mathscr{X}_{1}^{*}$, viewed as a subvariety of $\mathbb{C}^{g}$, is clearly invariant under the lattice subgroup generated by the columns of the matrix $(I, \Omega)$, the restriction of $\pi$ to $\mathscr{X}_{1}^{*}$ is a semiproper holomorphic mapping; so by the theorem of Kuhlmann and Whitney the image $\pi\left(\mathscr{X}_{1}^{*}\right)$ is a holomorphic subvariety of $\mathfrak{G}_{g}$. Now the subset of $\mathfrak{S}_{g}$ consisting of the normalized period matrices of compact Riemann surfaces is the image of a connected complex variety, Torelli space, under a generically two-to-one holomorphic mapping. Those points of Torelli space for which the image $\Omega$ is contained in $\pi\left(\mathscr{X}_{1}^{*}\right)$ form a holomorphic subvariety which omits the hyperelliptic Riemann surfaces by $\left(27^{\prime \prime}\right)$, so it must be a proper subvariety; thus the period matrix of a general Riemann surface is not contained in $\pi\left(\mathscr{X}_{1}^{*}\right)$, and that is just the desired result.

It follows from the preceding theorem and (27') that $W_{1}-W_{1}$ is at least an irreducible component of $X_{1}$ in the Jacobi variety of a general Riemann surface. This theorem and several other very interesting and suggestive results about the locus $X_{1}$ can be found in a preprint [17] by van Geemen and van der Geer, which only came to my attention after having essentially completed this paper. They show among other things that $W_{1}-W_{1}$ is an irreducible component of $X_{1}$ for any Riemann surface of genus $g \geqslant 5$ that is not either trigonal or a smooth plane curve of degree 5 , and that $X_{1} \cap\left(W_{2}-W_{2}\right)=W_{1}-W_{1}$ in the same cases.

7. For surfaces of sufficiently small genus, a good deal more can be said, and indeed said quite explicitly. Before turning to the consideration of these special cases, though, an auxiliary topological result should first be discussed. The homology classes in $H_{*}(J(M), \mathbb{Z})$ carried by the subvarieties $W_{r}$ of positive divisors have long been known and can be described quite explicitly; for many purposes a sufficiently explicit description is the intersection formula attributed to Poincaré, asserting that

$$
\Theta^{r} \sim r ! W_{g-r} \text { in } H_{2(g-r)}(J(M), \mathbb{Z}),
$$

where $\Theta^{r}=\Theta \cap \cdots \cap \Theta$ is the $r$-fold intersection homology class and denotes that the classes indicated are homologous. This formula is discussed in [18], among other places, and both there and in [3] can be found some results about the homology classes carried by the subvarieties of special positive divisors in some cases. What will be needed here though is rather the 
description of the homology class carried by $W_{1}-W_{1}$, a problem also considered by S. Diaz [8]; the result is the following.

THEOREM 4. In $H_{4}(J(M), \mathbb{Z})$,

$$
W_{1}-W_{1} \sim \begin{cases}W_{2} & \text { if } M \text { is hyperelliptic, } \\ 2 W_{2} & \text { if } M \text { is not hyperelliptic. }\end{cases}
$$

PRoof. Since there is no torsion in the homology of a torus it is enough to prove the desired result for homology with real coefficients, so by de Rham's theorem it is enough to show that $\int_{W_{1}-W_{1}} \xi=\eta \int_{W_{2}} \xi$ for every differential form $\xi$ of degree 4 on $J(M)$, where $\eta=1$ if $M$ is hyperelliptic and $\eta=2$ otherwise. Consider then the holomorphic mappings $\phi, \psi: M \times M \rightarrow J(M)$ defined by

$$
\phi\left(p_{1}, p_{2}\right)=w\left(p_{1}\right)+w\left(p_{2}\right), \quad \psi\left(p_{1}, p_{2}\right)=w\left(p_{1}\right)-w\left(p_{2}\right) .
$$

Outside a proper subvariety of $M \times M$ the mapping $\phi$ is a two-to-one mapping from $M \times M$ to $W_{2}$, while outside another proper subvariety of $M \times M$ the mapping $\psi$ is a two-to-one mapping if $M$ is hyperelliptic and a one-to-one mapping otherwise, as demonstrated in [20] for instance; therefore

$$
\int_{W_{2}} \xi=\frac{1}{2} \int_{M \times M} \phi^{*}(\xi) \text { and } \int_{W_{1}-W_{1}}=\frac{\eta}{2} \int_{M \times M} \psi^{*}(\xi)
$$

for every differential form $\xi$ of degree 4 on $J(M)$. Now there is a basis $\left\{\xi_{j}\right\}$ for the real differential forms of degree 1 on $J(M)$ such that

$$
w^{*}\left(\xi_{j}\right)= \begin{cases}\alpha_{j} & \text { if } 1 \leqslant j \leqslant g, \\ \beta_{j} & \text { if } g+1 \leqslant j \leqslant 2 g,\end{cases}
$$

where $\alpha_{j}, \beta_{j}$ are differential forms on $M$ dual to the corresponding generators of the fundamental group (hence of the first homology group) of $M$ and $w$ : $M \rightarrow J(M)$ is the imbedding (2); that is a simple consequence of the observation that the mapping $w$ takes the paths $\alpha_{j}, \beta_{j}$ to generators of the lattice subgroup $\mathscr{L}$. It follows readily from this that

$$
\begin{aligned}
\phi^{*}\left(\xi_{j}\right) & =\left\{\begin{array}{l}
\alpha_{j}\left(p_{1}\right)+\alpha_{j}\left(p_{2}\right), \\
\beta_{j}\left(p_{1}\right)+\beta_{j}\left(p_{2}\right),
\end{array}\right. \\
\psi^{*}\left(\xi_{j}\right) & = \begin{cases}\alpha_{j}\left(p_{1}\right)-\alpha_{j}\left(p_{2}\right) & \text { if } 1 \leqslant j \leqslant g, \\
\beta_{j}\left(p_{1}\right)-\beta_{j}\left(p_{2}\right) & \text { if } g+1 \leqslant j \leqslant 2 g,\end{cases}
\end{aligned}
$$

where $\alpha_{j}\left(p_{1}\right)$ is the differential form $\alpha_{j}$ on the first factor of the product $M \times M$. The exterior products of differential forms of degree 1 span the space of differential forms on the torus $J(M)$, so the proof finally reduces just to showing that

$$
\begin{aligned}
\int_{M \times M} \phi^{*}\left(\xi_{j}\right) & \wedge \phi^{*}\left(\xi_{k}\right) \wedge \phi^{*}\left(\xi_{l}\right) \wedge \phi^{*}\left(\xi_{m}\right) \\
& =\int_{M \times M} \psi^{*}\left(\xi_{j}\right) \wedge \psi^{*}\left(\xi_{k}\right) \wedge \psi^{*}\left(\xi_{l}\right) \wedge \psi^{*}\left(\xi_{m}\right) .
\end{aligned}
$$


Using (30), together with the observation from [19] or elsewhere that $\int_{M} \alpha_{j} \wedge \beta_{j}$ $=1$, while

$$
\int_{M} \alpha_{j} \wedge \alpha_{k}=\int_{M} \alpha_{j} \wedge \beta_{k}=\int_{M} \beta_{j} \wedge \beta_{k}=0 \text { for } j \neq k,
$$

it is readily verified that the two sides of (31) are equal, as desired, thereby concluding the proof. It might just be remarked in addition, though, that $W_{1}-W_{1}=W_{2}-E$ for a hyperelliptic surface $M$, as already noted, and since $W_{2}-E \sim W_{2}$, that provides an alternative proof of the desired result in the hyperelliptic case.

There are, of course, the natural extensions of the preceding result, although they are probably of somewhat less interest; but it may be of interest to see at least a sketch of the general result.

THEOREM $4^{\prime}$. In $H_{2 r}(J(M), \mathbb{Z})$ for $2 r \leqslant g$,

$$
W_{r}-W_{r} \sim \begin{cases}W_{2 r} & \text { if } M \text { is hyperelliptic, } \\
\left(\begin{array}{c}
2 r \\
r
\end{array}\right) W_{2 r} & \text { if } M \text { is not hyperelliptic. }\end{cases}
$$

Proof. If $M$ is hyperelliptic then $-W_{r}=W_{r}-r E$, so $W_{r}-W_{r}=W_{2 r}-E$ $\sim W_{2 r}$ as desired; therefore it can be supposed for the remainder of the proof that $M$ is not hyperelliptic. The holomorphic mapping $\phi: M^{2 r} \rightarrow W_{2 r}$, defined by

$$
\phi\left(p_{1}, \ldots, p_{2 r}\right)=\sum_{j=1}^{2 r} w\left(p_{j}\right)
$$

is of degree (2r)!, as demonstrated in [20] for example, and if it can be shown that the holomorphic mapping $\psi: M^{2 r} \rightarrow W_{r}-W_{r}$, defined by

$$
\psi\left(p_{1}, \ldots, p_{2 r}\right)=\sum_{j=1}^{r}\left(w\left(p_{j}\right)-w\left(p_{j+r}\right)\right),
$$

is of degree $(r !)^{2}$, then the argument used in the proof of Theorem 4 will lead directly to the desired result. Thus it is only necessary to show that for general points $p_{j}$ the only points $q_{j}$ for which $\psi\left(p_{1}, \ldots, p_{2 r}\right)=\psi\left(q_{1}, \ldots, q_{2 r}\right)$ are those such that $q_{1}, \ldots, q_{r}$ is some permutation of $p_{1}, \ldots, p_{r}$ and $q_{r+1}, \ldots, q_{2 r}$ is some permutation of $p_{r+1}, \ldots, p_{2 r}$. If that is not the case then for arbitrary points $p_{j}$ there must exist some points $q_{j}$ that are not such a permutation of the points $p_{j}$ but for which $\psi\left(p_{1}, \ldots, p_{2 r}\right)=\psi\left(q_{1}, \ldots, q_{2 r}\right)$, hence for which

$$
\sum_{j=1}^{r}\left(w\left(p_{j}\right)+w\left(q_{j+r}\right)\right)=\sum_{j=1}^{r}\left(w\left(p_{j+r}\right)+w\left(q_{j}\right)\right) ;
$$

that means that the point $t \in J(M)$ described by (32) actually lies in $W_{2 r}^{1}$. Fixing the points $p_{1}, p_{r+1}$, to any point $t \in W_{2 r}^{1}-W_{2 r}^{2}$ there corresponds a unique branched covering mapping $\pi_{t}: M \rightarrow \mathbb{P}^{1}$ of degree $2 r$, so to $t$ can be associated the divisors $\pi_{t}^{-1}\left(\pi_{t}\left(p_{1}\right)\right)$ and $\pi_{t}^{-1}\left(\pi_{t}\left(p_{1+r}\right)\right)$ of degree $2 r$ in $M$, and they must be holomorphic functions of $t$. Since the points $p_{2}, \ldots, p_{r}$ in the first 
divisor and $p_{r+2}, \ldots, p_{2 r}$ in the second divisor are quite arbitrary, it must be the case that $\operatorname{dim} W_{2 r}^{1} \geqslant 2 r-2$; but as shown in [20], among other places, that means the surface $M$ is hyperelliptic, contrary to assumption, so it is impossible. If $t \in W_{r}^{\nu}$ for all points $p_{j}$ and some $\nu>1$, the situation can be reduced to one for a smaller value of $r$, so that suffices for the sketch of the proof.

8. The simplest nontrivial case of the preceding considerations is that of a Riemann surface of genus $g=3$. In this case the vector of second-order theta functions describes a holomorphic mapping $\theta_{2}: \mathbb{C}^{3} \rightarrow \mathbb{C}^{8}$ or, equivalently, a holomorphic mapping $\left[\theta_{2}\right]: J(M) \rightarrow \mathbb{P}^{7}$, and $\operatorname{dim} S_{1}=7$ so that $\operatorname{dim} S_{1}^{\perp}=1$; thus $S_{1}^{\perp}$ is the span of a single vector $\alpha \in \mathbb{C}^{8}$, where $\alpha$ is any vector for which

$$
{ }^{\mathrm{t}} \alpha \cdot \theta_{2}(0)={ }^{\mathrm{t}} \alpha \cdot \partial_{j_{1} j_{2}} \theta_{2}(0)=0 \text { for } 1 \leqslant j_{1} \leqslant j_{2} \leqslant 3 \text {, }
$$

and $\left[S_{1}^{\perp}\right]=[\alpha]$ is a single point of $\mathbb{P}^{7}$. By Theorem 1 either $[\alpha] \notin K(\Omega)$ and $\Theta^{1}=\varnothing$, or $[\alpha] \in K(\Omega)$ and $\Theta^{1}$ is the single point $\Theta^{1}=\theta_{2}^{-1}([\alpha]) \in J(\Omega)$. The second case is that of a hyperelliptic Riemann surface, since, as is well known and discussed in [20], among other places, a hyperelliptic Riemann surface can be characterized by the condition that $\operatorname{dim} \Theta^{1}=g-3$; moreover, for a hyperelliptic Riemann surface it follows directly from (3) that $W_{g-1}^{1}=E$, hence by (10) that $\Theta^{1}=E-R$. Now if $\Theta^{1}$ is a single point, that point by symmetry must be the origin, so $\alpha=\theta_{2}(0)$; thus hyperelliptic Riemann surfaces can be characterized by the condition that

$$
{ }^{\mathrm{t}} \theta_{2}(0) \cdot \theta_{2}={ }^{\mathrm{t}} \theta_{2}(0) \cdot \partial_{j_{1} j_{2}} \theta_{2}(0)=0
$$

and on such a surface the hyperelliptic point $E$ and the Riemann point $R$ coincide. This characterization of hyperelliptic Riemann surfaces of genus 3 is neither new nor surprising, though, for by using Weierstrass's formula (4) it is readily seen to be equivalent to the characterization by the conditions that $\operatorname{dim} \Theta^{1} \geqslant 0$. Rather more interesting is the observation that in this case (19) takes the form $X_{1}=\left\{w \in J(M):{ }^{\mathrm{t}} \alpha \cdot \theta_{2}(w)=0\right\}$. The second-order theta function ${ }^{\mathrm{t}} \alpha \cdot \theta_{2}(w)$ transforms under the lattice $\mathscr{L}$ like a product of two first-order theta functions, from which it follows that the divisor of this function ${ }^{\mathrm{t}} \alpha \cdot \theta_{2}(w)$ in $J(M)$ carries the homology class $2 \Theta \sim 2 W_{2}$ in $H_{4}(J(M), \mathbb{Z})$. If $M$ is not hyperelliptic then $2 W_{2} \sim W_{1}-W_{1}$ by Theorem 4 , and since $W_{1}-W_{1} \subseteq X_{1}$, it must be the case that $W_{1}-W_{1}=X_{1}$ and the function ${ }^{\mathrm{t}} \alpha \cdot \theta_{2}(w)$ vanishes to first order on this locus. On the other hand if $M$ is hyperelliptic then $W_{2} \sim W_{1}-W_{1}$, but since $W_{1}-W_{1}=X_{1}$ in this situation anyway ${ }^{\mathrm{t}} \alpha \cdot \theta_{2}(w)$ vanishes precisely to the second order on this locus. At least it is true in any case that $X_{1}=W_{1}-W_{1}$. Finally, if $M$ is not hyperelliptic the whole construction leading to the space $Y_{1}$ is vacuous, so nothing further can be said. On the other hand if $M$ is hyperelliptic then, since $\left[T_{1}^{\perp}\right]$ is a nonempty subset of the point $\left[S_{1}^{\perp}\right.$ ], it must be the case that $T_{1}^{\perp}=S_{1}^{\perp}$, hence $T_{1}=S_{1}$ and $Y_{1}=X_{1}$; Theorem 2 then shows that $W_{1}-W_{1}=X_{1}=Y_{1}=\Theta$, although the fact that $W_{1}-W_{1}=\Theta$ in this case also follows directly since $E=-R$.

Both more interesting and more complicated is the case of a Riemann surface of genus $g=4$. In this case the vector of second-order theta functions 
describes a holomorphic mapping $\theta_{2}: \mathbb{C}^{4} \rightarrow \mathbb{C}^{16}$, and $\operatorname{dim} S_{1}=11$ so $\operatorname{dim} S_{1}^{\perp}$ $=5$; thus $S_{1}^{\perp}$ is the span of any five linearly independent vectors $\alpha_{1}, \ldots, \alpha_{5}$ in $\mathbb{C}^{16}$ for which

$$
{ }^{\mathrm{t}} \alpha_{i} \theta_{2}(0)={ }^{\mathrm{t}} \alpha_{i} \cdot \partial_{j_{1} j_{2}} \theta_{2}(0)=0 \text { for } 1 \leqslant j_{1} \leqslant j_{2} \leqslant 4
$$

It is known, as discussed in [34] for example, that there are just three possibilities for the subvariety $\Theta^{1}$ :
(i) $\Theta^{1}=W_{1}+E-R \quad$ when $M$ is hyperelliptic,
(ii) $\Theta^{1}=A \cup-A \quad$ for some points $A \neq-A$ in $J(M)$,
(iii) $\Theta^{1}=A$
for some point $A$ of order two in $J(M)$;

the general situation is (ii), while (iii) is the special case in which the two points $A$ and $-A$ coincide. If $M$ is hyperelliptic, so that $\Theta^{1}$ is the irreducible one-dimensional subvariety of $J(M)$ given by (i), then it follows readily from Theorem 2 that $Y_{1}=\left(W_{2}-E\right) \cup\left(W_{2}+2 E-2 R\right)$; but it was also noted earlier that $Y_{1}=W_{1}-W_{1}$ and is, hence, irreducible in this case, so $Y_{1}=W_{2}$ $-E=W_{2}+2 E-2 R$ and $3 E=2 R$ in $J(M)$. It also follows readily from Theorem 2 that $Y_{1}=(\Theta+A) \cup(\Theta-A)$ in case (ii) and $Y_{1}=\Theta+A$ in case (iii); in both cases, $\operatorname{dim} Y_{1}=3$. On the other hand, by definition, the subvariety $X_{1}$ can be described as

$$
X_{1}=\left\{w \in J(M):{ }^{\mathrm{t}} \alpha_{i} \cdot \theta_{2}(w)=0 \text { for } 1 \leqslant i \leqslant 5\right\}
$$

This subvariety could only be three-dimensional if the five second-order theta functions ${ }^{\mathrm{t}} \boldsymbol{\alpha}_{i} \cdot \theta_{2}(w)$ had a common nontrivial factor $f(w)$, and since $X_{1} \subseteq Y_{1}$ that factor could be taken to be of the form $f(w)=\theta(w-B)$ for some point $B \in J(M)$; but by (14) the quotients ${ }^{\mathrm{t}} \alpha_{i} \cdot \theta_{2}(w) / \theta(w-B)$ would all have to be constant multiples of $\theta(w+B)$, and it would be impossible for the functions ${ }^{\mathrm{t}} \boldsymbol{\alpha}_{i} \cdot \boldsymbol{\theta}_{2}(w)$ to be linearly independent. Thus $\operatorname{dim} X_{1}=2$ for any Riemann surface of genus $g=4$, and there are such surfaces for which $X_{1} \neq Y_{1}$, indeed for which these subvarieties have different dimensions. This is a rather special case though, arising from the trivial representation of the theta locus as a sum of subvarieties of complementary dimension in the form $\Theta=(\Theta-A)+A$; for surfaces of higher genus the corresponding representation of the theta locus would be a nontrivial one, if it existed at all. It should be noted that this does not show that $X_{1}=W_{1}-W_{1}$, but only that $W_{1}-W_{1}$ is an irreducible component of $X_{1}$. However, at least in case (ii) it follows that $W_{1}-W_{1} \subseteq X_{1} \subset Y_{1}=(\Theta+A) \cup(\Theta-A)$, and since $W_{1}-W_{1}$ is irreducible, it must be that either $W_{1}-W_{1} \subset \Theta+A$ or $W_{1}-W_{1} \subseteq \Theta-A$; but by symmetry if $W_{1}-W_{1} \subset \Theta+A$ then $W_{1}-W_{1} \subset \Theta-A$ as well, hence actually $W_{1}-W_{1} \subseteq(\Theta+A) \cap(\Theta-A)$. Further, though, the homology class of the intersection $(\Theta+A) \cap(\Theta-A)$ in $H_{4}(J(M), \mathbb{Z})$ is $\Theta^{2} \sim 2 W_{2}$ recalling (29), while $W_{1}-W_{1} \sim 2 W_{2}$ as well by Theorem 4; consequently $W_{1}-W_{1}=(\Theta+A) \cap(\Theta-A)$. 
9. The trisecant formula of Fay has extensions that lead to somewhat finer results about the locus $X_{1}$ as well as to quite interesting further properties of the second-order theta functions. These generalizations were discussed in [23], and the relevant material will be briefly summarized here. First, introducing the natural linear projection operator $P: \mathbb{C}^{g} \rightarrow \mathbb{C}^{G} / S_{1}$, there are vectors $\xi_{j k l} \in \mathbb{C}^{G} / S_{1}$ that are skew-symmetric in the indices $j, k, l$ and for which

$$
P \partial_{j} \theta_{2}\left(w\left(p_{1}\right)-w\left(p_{2}\right)\right)=q^{2} \sum_{k l} \xi_{j k l} w_{k}^{\prime}\left(p_{1}\right) w_{l}^{\prime}\left(p_{2}\right)
$$

for any points $p_{1}, p_{2} \in M$; here $q$ is a complex number that depends, of course, not just on the points $p_{1}, p_{2}$, but also on the path from $p_{2}$ to $p_{1}$ chosen in evaluating the left-hand side of (34), and on the choice of local coordinates near $p_{1}, p_{2}$ in terms of which the differentiations on the right-hand side of (34) are carried out, just as in Fay's formula (26). Although it was not needed before, it will later be necessary to have observed that $q \neq 0$ whenever $p_{1} \neq p_{2}$ on $M$. The vectors $\xi_{j k l}$ also have the property that

$$
\frac{1}{6} \sum_{k l m} P \partial_{j k l m} \theta_{2}(0) w_{k}^{\prime}(p) w_{l}^{\prime}(p) w_{m}^{\prime}(p)=\sum_{k l} \xi_{j k l} w_{k}^{\prime \prime}(p) w_{l}^{\prime}(p)
$$

for all points $p \in M$ and any index $j$. Next, letting $S_{1}^{*}$ be the subspace of $\mathbb{C}^{G}$ spanned by $S_{1}$ and any vectors in $\mathbb{C}^{G}$ representing the vectors $\xi_{j k l} \in \mathbb{C}^{G} / S_{1}$, and introducing the natural linear projection operator $P^{*}: \mathbb{C}^{G} \rightarrow \mathbb{C}^{G} / S_{1}^{*}$, there are vectors $\tau_{j k l m} \in \mathbb{C}^{G} / S_{1}^{*}$ that satisfy the symmetry conditions $\tau_{j k l m}=\tau_{k j l m}=$ $\tau_{j k m l}=\tau_{l m j k}$ and for which

$$
P^{*} \partial_{j_{1} j_{2}} \theta_{2}\left(w\left(p_{1}\right)-w\left(p_{2}\right)\right)=q^{2} \sum_{k l} \tau_{j_{1} j_{2} k l} w_{k}^{\prime}\left(p_{1}\right) w_{l}^{\prime}\left(p_{2}\right)
$$

for any points $p_{1}, p_{2} \in M$, the complex number $q$ being as in (34). These vectors $\tau_{j k l m}$ have, in addition, a number of other properties of some interest. First,

$$
\frac{1}{2} P^{*} \partial_{j k l m} \theta_{2}(0)=\tau_{j k l m}+\tau_{l j k m}+\tau_{k l j m}
$$

next

$$
\begin{aligned}
2 P^{*} \theta_{2}\left(w\left(p_{1}\right)\right. & \left.+w\left(p_{2}\right)-w\left(p_{3}\right)-w\left(p_{4}\right)\right) \\
& =Q \sum_{j k l m}\left(\tau_{j l k m}+\tau_{j m k l}\right) w_{j}^{\prime}\left(p_{1}\right) w_{k}^{\prime}\left(p_{2}\right) w_{l}^{\prime}\left(p_{3}\right) w_{m}^{\prime}\left(p_{4}\right)
\end{aligned}
$$

for all points $p_{j} \in M$ and for some complex number $Q$ depending on the points $p_{j}$ and the other choices made; and finally

$$
\sum_{l m} \tau_{j l k m} w_{l}^{\prime}(p) w_{m}^{\prime}(p)=0
$$

for all points $p \in M$ and all indices $j, k$.

The vectors $\xi_{j k l} \in \mathbb{C}^{G} / S_{1}$ now have a role to play in the investigation of the locus $X_{1} \subseteq J(M)$. First note that in view of their skew-symmetry there are really just $\left(\frac{\xi}{3}\right)$ of these vectors, and since

$$
\operatorname{dim} \mathbb{C}^{G} / S_{1} \geqslant\left(\begin{array}{l}
g \\
3
\end{array}\right) \text { whenever } g \geqslant 3
$$


there is no purely dimensional obstruction to these vectors being linearly independent.

THEOREM 5. If the vectors $\xi_{j k l}$ for $1 \leqslant j<k<l \leqslant g$ are linearly independent then $W_{1}-W_{1}$ is an irreducible component of $X_{1}$ and can only meet another irreducible component of $X_{1}$ at the point $O \in J(M)$.

Proof. The conclusion is automatically satisfied for hyperelliptic Riemann surfaces, so it can be supposed during the remainder of the proof that $M$ is not hyperelliptic. Since $P \theta_{2}(w)=0$ precisely when $\theta_{2}(w) \in S_{1}$, the subvariety $X_{1} \subseteq J(M)$ can be defined by $X_{1}=\left\{w \in J(M): P \theta_{2}(w)=0\right\}$. To prove the theorem it is then clearly enough just to show that the Jacobian matrix of these defining equations $\theta_{2}(w)$ at any point of $W_{1}-W_{1}$ other than the origin is of rank $g-2$. Now at the point $w\left(p_{1}\right)-w\left(p_{2}\right) \in W_{1}-W_{1}$ the $j$ th column of that Jacobian matrix is expressed in terms of the vectors $\xi_{j k l}$ by (34), and if $w\left(p_{1}\right) \neq w\left(p_{2}\right)$ then $p_{1} \neq p_{2}$ and $q \neq 0$; so what is to be demonstrated is that there are at most 2 linearly independent vectors $c=\left(c_{1}, \ldots, c_{g}\right) \in \mathbb{C}^{g}$ for which

$$
\begin{aligned}
0 & =\sum_{j k l} \xi_{j k l} c_{j} w_{k}^{\prime}\left(p_{1}\right) w_{l}^{\prime}\left(p_{2}\right) \\
& =\sum_{j<k<l} \xi_{j k l} \operatorname{det}\left(\begin{array}{lll}
c_{j} & w_{j}^{\prime}\left(p_{1}\right) & w_{j}^{\prime}\left(p_{2}\right) \\
c_{k} & w_{k}^{\prime}\left(p_{1}\right) & w_{k}^{\prime}\left(p_{2}\right) \\
c_{l} & w_{l}^{\prime}\left(p_{1}\right) & w_{l}^{\prime}\left(p_{2}\right)
\end{array}\right) .
\end{aligned}
$$

Of course whenever $c$ is a linear combination of $w^{\prime}\left(p_{1}\right)$ and $w^{\prime}\left(p_{2}\right)$ then (40) holds trivially. On the other hand, $w^{\prime}\left(p_{1}\right)$ and $w^{\prime}\left(p_{2}\right)$ are linearly independent, and if there were a vector $c$ not dependent on $w^{\prime}\left(p_{1}\right)$ and $w^{\prime}\left(p_{2}\right)$ but for which (40) held, there would be a nontrivial linear relation among the vectors $\xi_{j k l}$, contradicting the assumed linear independence. That suffices to conclude the proof.

For the case in which the Riemann surface $M$ is not hyperelliptic, the proof actually demonstrated somewhat more than asserted in the statement of the theorem, namely the following.

COROLlaRY 1. If the surface $M$ is not hyperelliptic and the vectors $\xi_{j k l}$ for $1 \leqslant j<k<l \leqslant g$ are linearly independent then the defining equations $P \theta_{2}(w)$ generate the proper ideal of the subvariety $W_{1}-W_{1}$ at each of its points outside the origin.

If $M$ is hyperelliptic and $\xi_{j k l}$ are linearly independent (a situation that may well not arise however) the same argument shows that the defining equations $P \theta_{2}(w)$ generate the proper ideal of the subvariety $X_{1}=W_{1}-W_{1}=W_{2}-E$ at each of its points outside the subvariety $2 W_{1}-E$. This exceptional locus $2 W_{1}-E$ is the set of all those points $w\left(p_{1}\right)-w\left(p_{2}\right) \in W_{1}-W_{1}$ at which the vectors $w^{\prime}\left(p_{1}\right)$ and $w^{\prime}\left(p_{2}\right)$ are linearly dependent or, equivalently, for which either $p_{1}=p_{2}$ or $p_{1}=\tau p_{2}$, where $\tau: M \rightarrow M$ is the hyperelliptic involution, and is precisely the branch locus of the natural mapping $M \times M \rightarrow W_{1}-W_{1}$; 
all the points of this locus except for the origin are regular points of the variety $W_{1}-W_{1}$, but the Jacobian matrix of the defining equations $P \theta_{2}(w)$ cannot have maximal rank at any of these points.

The condition that the vectors $\xi_{j k l}$ be linearly independent can be expressed as a property of the constants $\partial_{j k l m} \theta_{2}(0)$ by means of equation (35). It is more interesting and informative though to proceed more indirectly, through the additional vectors $\tau_{j k l m}$, and for that purpose it is convenient first to express these vectors in an alternative manner. It is a well-known theorem of $\mathbf{M}$. Noether, as discussed thoroughly in [11] and other places, that among the products $w_{j}^{\prime} w_{k}^{\prime}$ of the Abelian differentials on $M$ there are $3 g-3$ linearly independent terms when $M$ is not hyperelliptic, but only $2 g-1$ linearly independent terms when $M$ is hyperelliptic. There are thus $h$ linearly independent complex symmetric matrices $C=\left\{c_{j k}\right\}$ for which $\Sigma_{j k} c_{j k} w_{j}^{\prime} w_{k}^{\prime}=0$, where

$$
h= \begin{cases}\left(\begin{array}{c}
g-2 \\
2
\end{array}\right) & \text { if } M \text { is not hyperelliptic } \\
\left(\begin{array}{c}
g-1 \\
2
\end{array}\right) & \text { if } M \text { is hyperelliptic; }\end{cases}
$$

let $C^{\alpha}=\left\{c_{j k}^{\alpha}\right\}$ for $1 \leqslant \alpha \leqslant h$ be some basis for the space of such matrices. Introducing the symmetrized vectors

$$
\tilde{\tau}_{j k l m}=\tau_{j k l m}+\tau_{j m l k} \in \mathbb{C}^{G} / S_{1}^{*},
$$

it then follows from (39) that $\tilde{j}_{j k l m}=\sum_{\alpha} x_{j l}^{\alpha} c_{k m}^{\alpha}$ for some vectors $x_{j l}^{\alpha} \in \mathbb{C}^{G} / S_{1}^{*}$, and the symmetry properties of the vectors $\tau_{j k l m}$ easily imply that

$$
\sum_{\alpha} x_{j l}^{\alpha} c_{k m}^{\alpha}=\sum_{\alpha} x_{k m}^{\alpha} c_{j l}^{\alpha}
$$

Viewing the constants $c_{j l}^{\alpha}$ as forming an

$$
h \times\left(\begin{array}{c}
g+1 \\
2
\end{array}\right)
$$

complex matrix $C$, and the $i$ th components of the vectors $x_{j l}^{\alpha}$ as forming correspondingly a complex matrix $X_{i}$, this symmetry condition can be written as the matricial identities ${ }^{\mathrm{t}} X_{i} C={ }^{\mathrm{t}} C X_{i}$; from this and the observation that rank $C=h$, it follows quite readily that $X_{i}=A_{i} C$ for some symmetric $h \times h$ complex matrices $A_{i}=\left\{a_{\alpha \beta}^{i}\right\}$ and, consequently,

$$
\tilde{\tau}_{j k l m}=\sum_{\alpha \beta} a_{\alpha \beta} c_{j l}^{\alpha} c_{k m}^{\beta}
$$

for some vectors $a_{\alpha \beta}=a_{\beta \alpha} \in \mathbb{C}^{G} / S_{1}^{*}$ for $1 \leqslant \alpha, \beta \leqslant h$. Note that by using (43) the result of (37) can be rewritten as

$$
P^{*} \partial_{j k l m} \theta_{2}(0)=\sum_{\alpha \beta} a_{\alpha \beta}\left[c_{j k}^{\alpha} c_{l m}^{\beta}+c_{j l}^{\alpha} c_{k m}^{\beta}+c_{j m}^{\alpha} c_{k l}^{\beta}\right]
$$


and, correspondingly, the result of (38) can be written as

$$
\begin{aligned}
& P^{*} \theta_{2}\left(w\left(p_{1}\right)+w\left(p_{2}\right)-w\left(p_{3}\right)-w\left(p_{4}\right)\right) \\
& =\frac{1}{2} Q \sum_{\alpha \beta j k l m} a_{\alpha \beta} c_{j k}^{\alpha} c_{l m}^{\beta} w_{j}^{\prime}\left(p_{1}\right) w_{k}^{\prime}\left(p_{2}\right) w_{l}^{\prime}\left(p_{3}\right) w_{m}^{\prime}\left(p_{4}\right)
\end{aligned}
$$

for all points $p_{j} \in M$ and a suitable complex number $Q$.

Before continuing the argument it is possibly worth pausing briefly to make a few observations about these last few results. First the vectors $\xi_{j k l}$ can be expressed in terms of the partial derivatives of orders $0,2,4$ of the second-order theta functions at the origin as in (35), from which it appears to follow, as discussed in [23], that these vectors all lie in the subspace $S_{2} \subseteq \mathbb{C}^{G}$; but it is only the symmetrized versions (42) of the vectors $\tau_{j k l m}$ that can be so expressed, so while these symmetrized vectors $\tilde{\tau}_{j k l m}$ appear to lie in the subspace $S_{2} \subseteq \mathbb{C}^{G}$, it is by no means certain that the vectors $\tau_{j k l m}$ themselves do so. Second, and of much greater interest indeed, the expression (43) shows that the $\tilde{\tau}_{j k l m}$ must satisfy a great many more linear relations than just those imposed by the symmetry conditions; if the further auxiliary vectors $a_{\alpha \beta}$ for $1 \leqslant \alpha \leqslant \beta \leqslant h$ are linearly independent, then all the linear relations among the $\tilde{\tau}_{j k l m}$ are those following from the identity (43), and, correspondingly, all the linear relations among the vectors $P^{*} \partial_{j k l m} \theta_{2}(0)$ are generally those following from the identity (44). If the vectors $\xi_{j k l}$ are also linearly independent, then the vectors $P \partial_{j k l m} \theta_{2}(0)$ can be expressed uniquely as some linear combinations of the $\xi_{j k l}$ and suitable representatives of the $a_{\alpha \beta}$, and all the linear relations among the vectors $P \partial_{j k l m} \theta_{2}(0)$ are those following from such an identity; that must in particular be true for the linear relation

$$
\sum_{j k l m} P \partial_{j k l m} \theta_{2}(0) w_{j}^{\prime}(p) w_{k}^{\prime}(p) w_{l}^{\prime}(p) w_{m}^{\prime}(p)=0,
$$

which, as discussed in [23], is just a form of the well-known results of Dubrovin and others in which explicit solutions of the Kadomtsev-Petviashvili equation are given in terms of theta functions on Jacobi varieties, and should in principle be sufficient to determine whether there are any other such relations.

Now let $\nu$ be the dimension of the linear subspace of $\mathbb{C}^{G} / S_{1}$ spanned by all the vectors $\xi_{j k l}$, so that $0 \leqslant \nu \leqslant\left(\frac{\xi}{3}\right)$, and let $\mu$ be the dimension of the linear subspace of $\mathbb{C}^{G} / S_{1}^{*}$ spanned by all the vectors $a_{\alpha \beta}$, so that

$$
0 \leqslant \mu \leqslant\left(\begin{array}{c}
h+1 \\
2
\end{array}\right)
$$

where $h$ is given by (41). It might be expected that in general these dimensions would take their maximal values, so, in particular, Theorem 5 would apply in general. That is not the case for hyperelliptic Riemann surfaces though; indeed, noting that all of these vectors have representatives in $\mathbb{C}^{G}$ and recalling that

$$
\operatorname{dim} S_{1}=1+\left(\begin{array}{c}
g+1 \\
2
\end{array}\right)
$$


it follows immediately that

$$
\nu+\mu+1+\left(\begin{array}{c}
g+1 \\
2
\end{array}\right) \leqslant 2^{g},
$$

and when $\nu$ and $\mu$ have their maximal values even this inequality cannot hold for $g \leqslant 9$. Actually it was observed at the beginning of $\S 8$ that for a hyperelliptic surface of genus 3 the second-order theta function defining the locus $X_{1}=W_{1}-W_{1}$ vanishes to second order there, so $\nu=0$; thus it is not just the exceptionally large value of $h$ given by (41) that is the problem, since $\nu$ by itself does not have its maximal value $\nu=1$ in this case. For the hyperelliptic surfaces it was already shown in (27") that $X_{1}=W_{1}-W_{1}$, though, so this point will not be pursued further. On the other hand, at least for a nonhyperelliptic surface of sufficiently small genus, both $\nu$ and $\mu$ always take their maximal values.

THEOREM 6. The vectors $\xi_{j k l}$ for $1 \leqslant j<k<l \leqslant g$ and the vectors $a_{\alpha \beta}$ for $1 \leqslant \alpha \leqslant \beta \leqslant h$ are linearly independent for any nonhyperelliptic Riemann surface of genus $g=3,4$, or 5 .

Proof. It follows from (45) that $P^{*} \theta_{2}\left(W_{2}-W_{2}\right)$ is contained in the linear subspace of $\mathbb{C}^{G} / S_{1}^{*}$ spanned by the vectors $a_{\alpha \beta}$, hence $\theta_{2}\left(W_{2}-W_{2}\right)$ is contained in the linear subspace of $\mathbb{C}^{G}$ spanned by $S_{1}$ and representatives of the vectors $\xi_{j k l}$ and $a_{\alpha \beta}$; consequently, if $\delta_{2}$ is the dimension of the smallest linear subspace of $\mathbb{C}^{G}$ containing $\theta_{2}\left(W_{2}-W_{2}\right)$ then

$$
\nu+\mu+1+\left(\begin{array}{c}
g+1 \\
2
\end{array}\right) \geqslant \delta_{2} .
$$

When $g=3$ or 4 the subvariety $W_{2}-W_{2}$ is the entire Jacobi variety $J(M)$ so $\delta_{2}=G$. When $g=5, W_{2}-W_{2}$ is of codimension one in $J(M)$, and if $\delta_{2}<G$ the image of this subvariety under the imbedding $\theta_{2}: J(M) \rightarrow \mathbb{C}^{G}$ would lie in a hyperplane in $\mathbf{C}^{G}$, hence $W_{2}-W_{2}$ itself could be contained in the zero locus of some second-order theta function ${ }^{\mathrm{t}} c \cdot \theta_{2}(w)$; but $W_{2}-W_{2}$ is homologous to $6 W_{4}$ by Theorem $4^{\prime}$, while the divisor of any second-order theta function is only homologous to $2 \Theta \sim 2 W_{4}$, an evident impossibility. Thus when $g=5$ it is also the case that $\delta_{2}=G$. Using these explicit values for $\delta_{2}$, condition (48) can be rewritten as

$$
\nu+\mu \geqslant \begin{cases}1 & \text { when } g=3, \\ 5 & \text { when } g=4, \\ 16 & \text { when } g=5\end{cases}
$$

while a simple calculation from the formulas given for the maximum values of $\nu$ and $\mu$ shows that the reversed inequalities also hold; thus both $\nu$ and $\mu$ must take their maximum values in each of these cases, and that suffices to conclude the proof.

It is perhaps worth observing that the inequality (48) does hold universally, and that upon combining this with the obvious upper bounds for $\nu$ and $\mu$ there ensues the inequality $\delta_{2}<G$ whenever $M$ is not hyperelliptic and $g \geqslant 6$; the subvariety $\theta_{2}\left(W_{2}-W_{2}\right)$ must therefore lie in a proper linear subspace of $\mathbf{C}^{G}$ 
whenever $g \geqslant 6$ and $M$ is not hyperelliptic. The problem in extending the proof of Theorem 6 to surfaces of genus $g \geqslant 6$ is just that of determining $\delta_{2}$ in these cases. It is of course possible just to extend the analysis that led to the series of results (34) through (39) on which the proof of Theorem 2 was based, yielding at the same time relations between still higher-order derivatives of the secondorder theta functions and results about the higher-dimensional subvarieties $W_{r}-W_{r}$. This may indeed be a reasonably profitable direction for further investigation; the results become successively more complicated, but there may be enough overall regularity and simplicity to make them useful. At any rate it might be expected that the dimensions $\nu$ and $\mu$ always attain their maximal values for nonhyperelliptic surfaces, and that the imbedding dimension $\delta_{2}$ also always attains its maximal value so that (48) is an equality. It is a fairly simple matter to show that the analogous dimension $\delta_{1}$, the dimension of the smallest linear subspace of $\mathbb{C}^{G}$ containing $\theta_{2}\left(W_{1}-W_{1}\right)$, always attains its maximal value

$$
1+\left(\begin{array}{c}
g+1 \\
2
\end{array}\right)
$$

indeed, if that were not the case, then in view of (26) there would have to be a nontrivial linear relation

$$
c r\left(p_{1}, p_{2}\right)+q\left(p_{1}, p_{2}\right)^{2} \sum_{j \leqslant k} c_{j k} w_{j}^{\prime}\left(p_{1}\right) w_{k}^{\prime}\left(p_{2}\right)=0
$$

holding for all points $p_{1}, p_{2} \in M$, but that cannot happen, since for any fixed point $p_{1}$ the expressions $r\left(p_{1}, p_{2}\right)$ and $q\left(p_{1}, p_{2}\right)^{2} w_{k}^{\prime}\left(p_{2}\right)$ are, as noted in [23], linearly independent functions of the variable $p_{2} \in M$. Thus, at least in the case $\nu=1$, and quite possibly in all cases $\nu \geqslant 1$, when considering subvarieties defined in terms of the subspaces $S_{\nu} \subseteq \mathbb{C}^{G}$, there is no need to introduce auxiliary subspaces as was done in (20) when considering subvarieties defined in terms of the subspaces $S_{\nu}^{\perp} \subseteq \mathbb{C}^{G}$, so nothing playing the role of the subvarieties $Y_{\nu}$ could be expected. That may explain the observable asymmetry in the discussion in $\$ 5$.

Theorem 6 shows that the antic hypothesis and, hence, the conclusions of Theorem 5 and its corollary hold for all nonhyperelliptic surfaces of sufficiently small genus, leading thus to the following.

COROllary 2. The subvariety $W_{1}-W_{1}$ is an irreducible component of the locus $X_{1}$ for any Riemann surface of genus $g=3,4$, or 5; if, moreover, the Riemann surface is nonhyperelliptic then the defining equations $P_{2}(w)$ generate the proper ideal of $W_{1}-W_{1}$ at each of its points outside the origin.

For a surface of genus $g=3$ or for a hyperelliptic surface $X_{1}=W_{1}-W_{1}$, as already noted, while for a nonhyperelliptic surface of genus $g=4$, it is an easy consequence of the above corollary that $W_{1}-W_{1}$ is the unique irreducible component of $X_{1}$ of dimension $\geqslant 2$ passing through the origin; indeed, if there is another such component $A$ then, since $A$ and $W_{1}-W_{1}$ are two-dimensional 
subvarieties of the four-dimensional manifold $J(M)$, necessarily

$$
\operatorname{dim}\left(A \cap\left(W_{1}-W_{1}\right)\right)=0,
$$

and at any point of $A \cap\left(W_{1}-W_{1}\right)$ outside the origin the defining equations $P_{2}(w)$ describe the union $A \cup\left(W_{1}-W_{1}\right)$, contradicting the corollary.

There remain the questions whether the defining equations $\mathrm{P \theta}_{2}(w)$ generate the proper ideal of the subvariety $X_{1}$ at the origin for a nonhyperelliptic Riemann surface of genus $g=3,4$, or 5 , and whether $X_{1}$ can ever have any components other than $W_{1}-W_{1}$ for a surface of genus $g=4$ or 5 ; and there are other subvarieties of $J(M)$ and approaches to these questions suggested by the preceding discussion. Indeed, between the linear subspaces $S_{1}$ and $S_{2}$ there is the intermediate subspace $S_{1}^{*}$ spanned by $S_{1}$ and the vectors $\xi_{j k l}$, and that leads to a consideration of the subvariety

$$
X_{1}^{*}=\left\{w \in J(M): \theta_{2}(w) \in S_{1}^{*}\right\}=\left\{w \in J(M): P^{*} \theta_{2}(w)=0\right\},
$$

for which, of course, $X_{1} \subseteq X_{1}^{*} \subseteq X_{2}$. At least for surfaces of genus $g=4$ this subvariety can quite readily be analyzed by using equation (45), although this does require a further treatment of the dependence of the scalar parameter $Q$ on the choice of the points $p_{j}$; the result is that $X_{1}^{*}$ is a three-dimensional subvariety of $J(M)$ of the form $X_{1}^{*}=\left(W_{2}-V\right) \cup\left(V-W_{2}\right)$ for some curve $V \subseteq J(M)$, the zero locus of a particular second-order theta function. The analogue of (45), but with the projection operator $P$ in place of $P^{*}$, a formula to be found in [23], can be used to obtain alternative descriptions of the pull back of the locus $X_{1}$ to the product $M^{4}$, thus providing a somewhat different approach that will be discussed elsewhere; it may serve to describe the locus $X_{1}$ more fully, and at least leads to a rather interesting further analysis of the second-order theta functions. The extension of this approach to Riemann surfaces of higher genus and the subvarieties $X_{\nu}$ for $\nu>1$ requires a further exploration of yet higher-order derivatives of the second-order theta functions at the origin.

10. The derivation of the properties of the second-order theta functions as described in the preceding sections is obviously just a preliminary step towards a more complete theory of these fascinating functions, so a few words about where these directions of research might lead should possibly be added here. Probably the most interesting of the properties discussed here are those related to the linear independence of the auxiliary vectors $\xi_{j k l}$ and $\tau_{j k l m}$, which closely involve the derivatives of the second-order theta functions at the origin; such properties should lead to further results about the roles these theta functions play in leading to explicit solutions of certain nonlinear partial differential equations. There is clearly much work to be done in that direction, On the other hand the subvarieties $W_{\nu}-W_{\nu}$ are of considerable interest as being the most invariant among the subvarieties of positive divisors, being independent of choices of base points and firmly anchored at the origin in the Jacobi variety $J(M)$. These are the subvarieties most likely to be amenable to an explicit description in terms of the period matrix alone, through the intermediation of the theta functions; that is certainly quite satisfactorily the case for Riemann 
surfaces of genus $g=3$, and in at least a primitive manner for surfaces of genus $g=4$ and 5 , although clearly much remains to be done. The subvariety $W_{1}-W_{1}$ itself determines the Riemann surface $M$, being birationally equivalent to the symmetric product $M^{(2)}$ and having the property that $\left(W_{1}-W_{1}\right) \cap$ $W_{g-1}$ consists of $g$ copies of $\pm W_{1}$. That leads to yet another approach to Torelli's theorem that the canonical period matrix of a Riemann surface uniquely determines the surface, and more importantly leads to another approach to describing mappings between Riemann surfaces directly in terms of their period matrices.

There is again the question of what can be said about the second-order theta functions on general principally polarized Abelian varieties, as distinct from Jacobi varieties, and related to that the question of the extent to which these properties of the theta functions characterize the Jacobi varieties among arbitrary principally polarized Abelian varieties. As already noted, the condition that $\operatorname{dim} K(\Omega) \cap\left[S_{1}^{\perp}\right] \geqslant g-4$, as a condition on the period matrix $\Omega$, picks out a subvariety of the Siegel upper half-space, one component of which is the closure of the set of period matrices of Riemann surfaces of genus $g$; the condition that $\operatorname{dim} K(\Omega) \cap\left[S_{1}\right] \geqslant 2$ may have the same property, since at least a somewhat related condition described by Novikov has that property, as discussed earlier here. If both of these conditions are satisfied then the theta locus $\Theta$ contains the subvariety $X_{1}+\Theta^{1}$, and that may be sufficient with some generic regularity conditions to guarantee that the Abelian variety is a Jacobi variety; the condition $\Theta=X_{1}+\Theta^{1}$ should correspondingly characterize the Jacobi variety of a hyperelliptic Riemann surface. The condition $X_{1}+\Theta^{1} \subseteq \Theta$ should by itself suffice to show that $\operatorname{dim} X_{1} \leqslant 2$; the geometric consequences of such an inclusion would at any rate be an interesting topic to pursue further.

11. There are yet other approaches to using the second-order theta functions to describe canonical subvarieties of Jacobi varieties. Only one such, which if in some ways leads to less explicit descriptions nonetheless in other ways leads to more comprehensive descriptions, will be discussed here; it really involves the mapping $\theta_{2}^{*}: \mathbb{C}^{g} \rightarrow \mathbb{C}^{G}$ rather than $\theta_{2}: \mathbb{C}^{g} \rightarrow \mathbb{C}^{G}$, with notation as in $\S 4$, so it can be used to describe subvarieties $J(M)$ that are not necessarily symmetric under the involution $E: J(M) \rightarrow J(M)$ induced by the mapping $w \rightarrow-w$. If $p_{1}, \ldots, p_{n}$ are any $n$ distinct points of the Riemann surface $M$ and $\mathfrak{d}=p_{1}+$ $\cdots+p_{n}$ is the divisor formed from these points, then introduce the $2^{g} \times n$ matrix

$$
\boldsymbol{\Theta}_{2}(w ; \delta)=\left\{\boldsymbol{\theta}_{2}\left(w\left(p_{1}\right)+\frac{1}{2} w\right), \ldots, \theta_{2}\left(w\left(p_{n}\right)+\frac{1}{2} w\right)\right\} .
$$

The rank of this matrix is clearly the same at all points $w+\lambda$ as $\lambda$ ranges over the lattice $\mathscr{L}$, so any condition on $w$ expressed in terms of the rank of this matrix can be used to describe a well-defined subvariety of the torus $J(M)$. The basic result, as described in [21], is

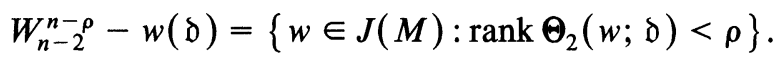

It is not really necessary that the points $p_{j} \in M$ be distinct if the appropriate conventions are followed, and indeed in [21] this description was really given 
primarily in the case in which all the points coincide. If, for example, $p_{1}=p_{2}$ then the column $\theta_{2}\left(w\left(p_{2}\right)+\frac{1}{2} w\right)$ must be replaced by

$$
\partial \theta_{2}\left(w(z)+\frac{1}{2} w\right) /\left.\partial z\right|_{z=p_{1}},
$$

while if $p_{1}=p_{2}=p_{3}$ then, in addition, the column $\theta_{2}\left(w\left(p_{3}\right)+\frac{1}{2} w\right)$ must be replaced by

$$
\partial^{2} \theta_{2}\left(w(z)+\frac{1}{2} w\right) /\left.\partial z^{2}\right|_{z=p_{1}}
$$

and so on; the derivatives are calculated in terms of any local coordinate $z$ near the point $p_{1}$ on $M$. This thus provides a complete description of all the subvarieties of special positive divisors in $J(M)$. In particular, for $n=\rho=3$,

$$
W_{1}-w(\delta)=\left\{w \in J(M): \operatorname{rank} \Theta_{2}(w ; \delta)<3\right\},
$$

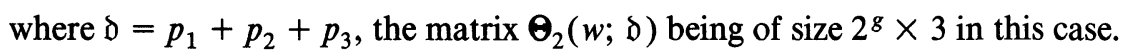

The principal disadvantage of this description is that it is necessary to know the images $w\left(p_{j}\right)$ of $n$ points of the Riemann surface under the canonical imbedding of that surface in its Jacobi variety, or the images of these points and the tangent vectors to the imbedded surface at these points if there are simple coincidences, and so on. Actually since there is full freedom of translation it is really only necessary to know the $n-1$ intrinsically determined vectors $w\left(p_{j}\right)-w\left(p_{n}\right)$ for $1 \leqslant j \leqslant n-1$ if all the points $p_{j}$ are distinct, or the $n-1$ vectors $d^{\nu} w(z) /\left.d z^{\nu}\right|_{z=p}$ for $1 \leqslant \nu \leqslant n-1$ if all of the points $p_{j}$ coincide with $p$. It does mean that it requires knowing considerably more than just the period matrix to use this method to describe the subvarieties of special positive divisors. That naturally leads to the question of what the corresponding locus is if in place of the points $w\left(p_{j}\right)$ simply $n$ arbitrary points $w_{j} \in J(M)$ are taken with the natural modifications in cases of coincidences. This question has so far only been treated in the special case (51), but the results there are reasonably complete and quite interesting and hold for more general Abelian varieties than just Jacobi varieties. The result established in [22] is that if $\Omega$ is an irreducible period matrix in the Siegel upper half-space, if $w_{1}, w_{2}, w_{3}$ are three points of $J(\Omega)$ in sufficiently general position, and if

$$
\boldsymbol{\theta}_{2}(w)=\left\{\theta_{2}\left(w_{1}+\frac{1}{2} w\right), \theta_{2}\left(w_{2}+\frac{1}{2} w\right), \theta_{3}\left(w_{3}+\frac{1}{2} w\right)\right\},
$$

then $\left\{w \in J(\Omega)\right.$ : $\left.\operatorname{rank} \Theta_{2}(w)<3\right\}$ either consists of finitely many points (indeed of at least three, but always a bounded number of points independent of the choice of $w_{j}$ ) or is a nonsingular irreducible curve $M$ of genus $g$ (in which case $J(\Omega)=J(M)$ is the Jacobi variety of that Riemann surface $M$ ). The condition that the points $w_{j}$ be in sufficiently general position can be given a very precise meaning, namely that there is no nonzero complex multiplication $F$ of $J(\Omega)$ for which $F\left(w_{1}\right)=F\left(w_{2}\right)=F\left(w_{3}\right)$. The corresponding result for coincident points $w_{j}$ was established by Welters in [40], and both results were generalized further and put into a more definitive form by Welters in [41]. These results provide a necessary and sufficient condition that an Abelian variety $J(\Omega)$ actually be a Jacobi variety, thus leading to another approach to the Schottky problem, as discussed in [22 and 41]; a related characterization for nonhyperelliptic Jacobi varieties was given by Welters in [42]. In [4] 
Arbarello and De Concini show that this condition can be expressed as a set of equations in the theta constants and their derivatives; this gives a condition, quite directly expressed in terms of explicit functions of the matrix $\Omega$, that $\Omega$ be the normalized period matrix of a Riemann surface. Some simplifications of this condition were discussed by Welters in [41]. The functions as functions of $\Omega$ seem nonetheless somewhat complicated insofar as their behavior under the action of the generalized modular group on the Siegel upper half-space. On the other hand, this approach appears able to shed some light on the status of a conjectured solution of the Schottky problem proposed by Novikov and to be complementary in some ways to another approach to the Schottky problem developed by Shiota, as discussed in [4].

\section{BIBLIOGRAPHY}

1. A. Andreotti, On a theorem of Torelli, Amer. J. Math. 80 (1958), 801-828.

2. A. Andreotti and A. L. Mayer, On period relations for abelian integrals on algebraic curves, Ann. Scuola Norm. Sup. Pisa 21 (1967), 189-238.

3. E. Arbarello et al, Topics in the theory of algebraic curves (to appear).

4. E. Arbarello and C. De Concini, On a set of equations characterizing Riemann matrices, Ann. of Math. (to appear).

5. A. Beauville, Prym varieties and the Schottky problem, Invent. Math. 41 (1977), 148-196.

6. L. Bers, Uniformization, moduli, and Kleinian groups, Bull. London Math. Soc. 4 (1972), 257-300.

7. F. Conforto, Abelsche Funktionen und algebraische Geometrie, Springer-Verlag, 1956.

8. S. Diaz, Exceptional Weierstrass points and the divisor on moduli space that they define, $\mathrm{Ph} . \mathrm{D}$. thesis, Brown Univ., 1982.

9. B. A. Dubrovin, Theta functions and nonlinear equations, Russian Math. Surveys 36 (1981), 11-92.

10. H. M. Farkas and H. E. Rauch, Theta functions with applications to Riemann surfaces, Williams \& Wilkins, Baltimore, Md., 1974.

11. H. M. Farkas and I. Kra, Riemann surfaces, Springer-Verlag, 1981.

12. H. M. Farkas, On Fay's trisecant formula (preprint).

13. J. D. Fay, Theta functions and Riemann surfaces, Lecture Notes in Math., vol. 352, Springer-Verlag, 1973.

14. On the even-order vanishing of Jacobian theta functions, Duke Math. J. 51 (1984), $109-132$.

15. G. Fischer, Complex analytic geometry, Lecture Notes in Math., vol. 538, Springer-Verlag, 1976.

16. B. van Geemen, Siegel modular forms vanishing on the moduli space of curves, Preprint No. 294, Dept. Math., Univ. Utrecht, 1983.

17. B. van Geemen and G. van der Geer, Kummer varieties and moduli spaces of Abelian varieties, Preprint No. 308, Dept. Math., Univ. Utrecht, 1983.

18. P. Griffiths and J. Harris, Principles of algebraic geometry, Wiley, 1978.

19. R. C. Gunning, Lectures on Riemann surfaces, Princeton Univ. Press, Princeton, N.J., 1966.

20. , Lectures on Riemann surfaces: Jacobi varieties, Princeton Univ. Press, Princeton, N.J., 1972.

21. __ On generalized theta functions, Amer. J. Math. 104 (1982), 183-208.

22. __ Some curves in Abelian varieties, Invent. Math. 66 (1982), 377-389.

23. __ Some identities for Abelian integrals, Amer. J. Math. 108 (1985).

24. R. W. H. T. Hudson, Kummer's quartic surface, Cambridge Univ. Press, 1905.

25. J.-I. Igusa, Theta functions, Springer-Verlag, 1972.

26. A. Krazer, Lehrbuch der Thetafunktionen, Teubner, Leipzig, 1903.

27. A. Krazer and W. Wirtinger, Abelsche Funktionen und allgmeine Thetafunktionen, Enzykl. der Math. Wiss., II B7, 604-873. 
28. J. Lewittes, Riemann surfaces and the theta function, Acta Math. 111 (1964), 37-61.

29. H. H. Martens, A new proof of Torelli's theorem, Ann. of Math. (2) 78 (1963), 107-111.

30. $111-120 ; 233$ (1968), 89-100.

31. 394-408. , On the partial derivatives of thetafunctions, Comment. Math. Helv. 48 (1973),

32. T. Matsusaka, On a theorem of Torelli, Amer. J. Math. 80 (1958), 784-800.

33. A. Mayer, Special divisors and the Jacobian variety, Math. Ann. 153 (1964), 163-167.

34. D. Mumford, Curves and their Jacobians, Univ. of Michigan Press, Ann Arbor, Mich., 1975.

35. , Tata lectures on theta, vols. I, II, Birkhäuser, Boston, 1983, 1984.

36. M. Namba, Families of meromorphic functions on compact Riemann surfaces, Lecture Notes in Math., vol. 767, Springer-Verlag, 1979.

37. B. Riemann, Theorie der Abel'schen Funktionen, J. Reine Angew. Math. 54 (1857), 115-180; Ueber das Verschwinden der Theta-Funktionen, J. Reine Angew. Math. 65 (1865), 161-173.

38. C. L. Siegel, Topics in complex function theory, Vols. 1-3, Wiley, 1969.

39. A. Weil, Zum Beweis des Torellischen Satzes, Nachr. Akad. Wiss. Göttingen (1957), 33-53.

40. G. E. Welters, On flexes of the Kummer variety, Preprint, Facultad Mat. Univ. Barcelona, 1983.

41. __ A criterion for Jacobi varieties, Preprint, Facultad Mat. Univ. Barcelona, 1983.

42. A A characterization of non-hyperelliptic Jacobi varieties, Invent. Math. 74 (1983), 437-440.

43. H. Weyl, The concept of a Riemann surface, Addison-Wesley, Reading, Mass., 1964.

44. W. Wirtinger, Untersuchungen über Thetafunktionen, Teubner, Leipzig, 1895.

Department of Mathematics, Princeton University, Princeton, New Jersey 08544 